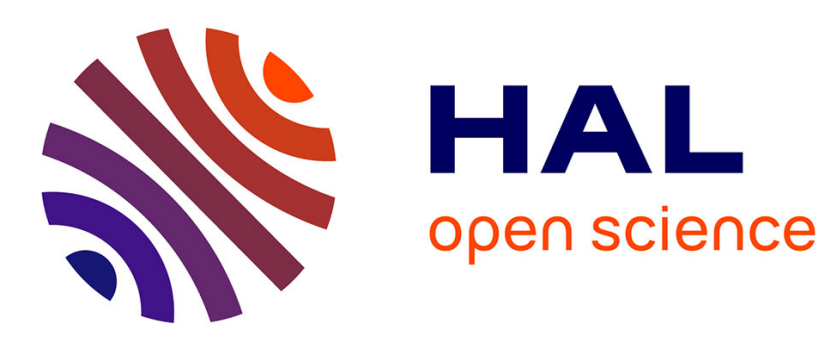

\title{
Study of the large-eddy simulation subgrid terms of a low Mach number anisothermal channel flow
}

Dorian Dupuy, Adrien Toutant, Françoise Bataille

\section{To cite this version:}

Dorian Dupuy, Adrien Toutant, Françoise Bataille. Study of the large-eddy simulation subgrid terms of a low Mach number anisothermal channel flow. International Journal of Thermal Sciences, 2019, 135, pp.221 - 234. 10.1016/j.ijthermalsci.2018.09.001 . hal-01906390

\section{HAL Id: hal-01906390 \\ https://hal-univ-perp.archives-ouvertes.fr/hal-01906390}

Submitted on 11 Feb 2021

HAL is a multi-disciplinary open access archive for the deposit and dissemination of scientific research documents, whether they are published or not. The documents may come from teaching and research institutions in France or abroad, or from public or private research centers.
L'archive ouverte pluridisciplinaire HAL, est destinée au dépôt et à la diffusion de documents scientifiques de niveau recherche, publiés ou non, émanant des établissements d'enseignement et de recherche français ou étrangers, des laboratoires publics ou privés. 


\title{
Study of the large-eddy simulation subgrid terms of a low Mach number anisothermal channel flow
}

\author{
Dorian Dupuy ${ }^{1}$, Adrien Toutant*1, and Françoise Bataille ${ }^{1}$ \\ ${ }^{1}$ PROMES CNRS, Université de Perpignan Via Domitia, Rambla de la \\ thermodynamique, Tecnosud, 66100 Perpignan, France
}

(Dated: 18 July 2018)

\begin{abstract}
The subgrid terms of the low Mach number equations are investigated in a strongly anisothermal low Mach number flow. The filtered low Mach number equations are established in three formulations in order to compare the unweighted classical filter and the density-weighted Favre filter on the one hand, and the filtering of the momentum conservation equation and the velocity transport equation on the other hand. In the three formulations, we establish the filtered equations of mass conservation, momentum conservation, energy conservation, of the ideal gas law and of the resolved kinetic energy transport equation. The magnitude of all subgrid terms is assessed a priori in the three formulations using the results of direct numerical simulations of a strongly anisothermal fully developed turbulent channel flow. The classification of the subgrid terms gives the relevance of various effects of the temperature gradient.
\end{abstract}

\section{Introduction}

In solar power towers, the solar flux is concentrated towards the solar receiver, wherethrough its energy is transferred to the heat transfer fluid. The optimisation of the internal geometry of the solar receiver is a key challenge for the efficiency of solar power towers. The thermal exchange towards the fluid should be maximised while the pressure loss should be minimised. This long-term goal would benefit from accurate numerical simulations of the low Mach number strongly anisothermal turbulent flows found in solar receivers [34]. However, high numerical costs prevent the direct numerical simulation (DNS) of all scales of turbulence in the conditions of a real solar receiver. An effective alternative is the thermal large-eddy simulation (LES). The LES resolves the largest scales of the flow and models the effect of the small scales on the large scales. The method requires subgrid-scale models suited to low Mach number strongly anisothermal turbulent flows $[4,5,16]$.

The subgrid terms can be investigated a priori from high-resolution three-dimensional flow fields. A priori tests were carried out with particle image velocimetry measurements $[24,10]$ and DNS results, in incompressible isotropic homogeneous turbulence

\footnotetext{
*Corresponding author : adrien.toutant@univ-perp.fr
} 
$[14,1]$, rotating turbulence [25], channel flows [30, 22], in two phase divergence-free flows [40], in passive and active scalar decaying homogeneous turbulence [12, 20] and in flows with purely compressible effects, in a temporal shear layer $[43,44,42]$ and in freely decaying homogeneous isotropic turbulence [26]. In flows with variable fluid properties, the analysis can be carried out using two large-eddy simulation filters, the unweighted classical filter and the density-weighted Favre filter [18]. The Favre filter is used by most authors in compressible flows [19]. The classical filter has been employed by Yoshizawa [46], Sun and Lu [37], Boersma and Lele [8], Bodony and Lele [7] and Sidharth et al. [36]. In addition, the set of subgrid terms to model depends on the formulation of the filtered governing equations, of the energy equation in particular but also of the momentum equation [35]. Using a priori tests, Vreman et al. [43] and Martín et al. [26] assessed the amplitude of all subgrid terms involved in the compressible Navier-Stokes equations for different formulations of the energy conservation equation.

In the literature, the subgrid terms have not been investigated for low Mach number strongly anisothermal turbulent flows. In this paper, we extend the analysis to this configuration. In strongly anisothermal flows, the large temperature differences require taking into account the variations of the fluid properties. If in addition the flow is turbulent, the variations of the fluid properties are related to the velocity fluctuations. The figure 2 is an illustration of the correlation between the velocity and temperature. The isosurface of figure 2 is not only an isotherm but also a surface of isodensity, isoviscosity and isoconductivity. Both the velocity and temperature profiles are turbulent and exhibit a wide range of length scales. The correlations between velocity and temperature lead to additional subgrid terms associated with the nonlinearities of the viscous term and of the heat flux. In the literature, these additional subgrid terms are always neglected (see for example [34, 45, 2, 11, 33, 32, 23]). However, to the best of our knowledge, there is no study to justify this assumption in low Mach number flows. We investigate whether these additional subgrid terms may be neglected. This study is essential for the flows found in solar receivers, characterised by a strong coupling between turbulence and temperature, along with high variations of the fluid properties (density, viscosity and thermal conductivity) with temperature [39].

We study the subgrid terms using the results of direct numerical simulations of a strongly anisothermal fully developed turbulent channel flow. The investigation is based on a particular form of the Navier-Stokes equations under the low Mach number hypothesis, called low Mach number equations [15]. The filtering of the low Mach number equations gives rise to specific subgrid terms. Three formulations of the filtered low Mach number equations are investigated, which leads to three specific sets of subgrid terms. The analysis is carried out using the classical filter and the Favre filter. With the classical filter, we compare the filtering of the momentum conservation equation to the filtering of the velocity transport equation. We assess the magnitude of each term in the three formulations. This provides insights on the asymmetry of the subgrid and non-subgrid terms between the two sides of the channel and on the relative importance of the various competing physical processes. The study takes into account both the subgrid terms associated with non-commutation of 
the filter with the derivative and the nonlinearities arising from the large variations of the fluid properties. The objective is to identify the subgrid terms that can be neglected and the subgrid terms that should be modelled in the three formulations.

We describe the low Mach number equations in section 2. In section 3, we study the filtering of the low Mach number equations in three formulations. The subgrid terms derived in the general case are then estimated in the channel flow configuration. The detailed channel flow configuration can be found in section 4 as well as the numerical method used to compute the subgrid terms. The results are discussed in section 5 .

\section{Low Mach number equations}

We consider a turbulent flow at low Mach number $(M a<0.3)$. The low Mach number hypothesis let us use Paolucci's method [29] to remove acoustic effects from the Navier-Stokes equations. Each variable of the Navier-Stokes equations is written as a power series of the squared Mach number. Keeping only the smaller-order terms leads to the so-called low Mach number equations. The pressure is split in two parts: the thermodynamical pressure $P$, mean pressure in the domain, and the mechanical pressure $P_{0}$, associated with momentum variations. The thermodynamical pressure is constant in space. The gas is air, an ideal gas for the purpose of this study. The effects of gravity are neglected.

Those considerations lead to the low Mach number equations, given by:

- Mass conservation equation

$$
\frac{\partial \rho}{\partial t}+\frac{\partial \rho U_{j}}{\partial x_{j}}=0
$$

- Momentum conservation equation

$$
\frac{\partial \rho U_{i}}{\partial t}=-\frac{\partial \rho U_{j} U_{i}}{\partial x_{j}}-\frac{\partial P}{\partial x_{i}}+\frac{\partial \Sigma_{i j}}{\partial x_{j}}
$$

- Energy conservation equation

$$
\frac{\partial U_{j}}{\partial x_{j}}=-\frac{1}{\gamma P_{0}}\left[(\gamma-1) \frac{\partial Q_{j}}{\partial x_{j}}-\frac{\partial P_{0}}{\partial t}\right],
$$

- Ideal gas law

$$
T=\frac{P_{0}}{\rho r},
$$

with $\rho$ the density, $T$ the temperature, $\Sigma_{i j}$ the shear-stress tensor, $Q_{j}$ the conductive heat flux, $\gamma$ the heat capacity ratio, $r$ the ideal gas specific constant, $t$ the time, $P$ the mechanical pressure, $P_{0}$ the thermodynamical pressure, $U_{i}$ the $i$-th component 
of velocity and $x_{i}$ the Cartesian coordinate in $i$-th direction. Einstein summation convention is used and $\delta_{i j}$ is the Kronecker delta.

In the low Mach number equations, the energy conservation equation has a distinctive form. The local conservation of energy is imposed by a constraint on the divergence of the velocity. Namely, the dilatation of the fluid is proportional to the difference between the conductive heat flux and the global variation of thermodynamical pressure. To obtain equation (3), we use the low Mach number hypothesis to approximate the compressible energy conservation equation in enthalpy form as [28]

$$
\frac{\partial \rho C_{p} T}{\partial t}+\frac{\partial \rho U_{j} C_{p} T}{\partial x_{j}}=\frac{\partial P_{0}}{\partial t}-\frac{\partial Q_{j}}{\partial x_{j}}
$$

where $C_{p}$ is the heat capacity at constant pressure. We then use the ideal gas law (4) to substitute in this equation $\rho T$ with $P_{0} / r$. Using the fact that the thermodynamical pressure is constant in space, we isolate the divergence of the velocity and combine the two temporal terms.

We assume that air is a Newtonian fluid to compute the shear-stress tensor,

$$
\Sigma_{i j}=\mu(T)\left(\frac{\partial U_{i}}{\partial x_{j}}+\frac{\partial U_{j}}{\partial x_{i}}\right)-\frac{2}{3} \mu(T) \frac{\partial U_{k}}{\partial x_{k}} \delta_{i j}
$$

The heat flux is given by

$$
Q_{j}=-\lambda(T) \frac{\partial T}{\partial x_{j}}
$$

The variations of viscosity with temperature are accounted for by the Sutherland's law [38],

$$
\mu(T)=\mu_{0}\left(\frac{T}{T_{0}}\right)^{\frac{3}{2}} \frac{T_{0}+S}{T+S},
$$

with $\mu_{0}=1.716 \cdot 10^{-5}, S=110.4$ and $T_{0}=273.15 \mathrm{~K}$. The thermal conductivity is deduced from the Prandlt number $\operatorname{Pr}$ and the heat capacity at constant pressure $C_{p}$, both assumed constant with $\operatorname{Pr}=0.76$ and $C_{p}=1005 \mathrm{~J} \mathrm{~kg}^{-1} \mathrm{~K}^{-1}$. The ideal gas specific constant is $r=287 \mathrm{~J} \mathrm{~kg}^{-1} \mathrm{~K}^{-1}$.

We will study the filtering of the low Mach number equations in three formulations that we shall call the Classical formulation, the Favre formulation and the Velocity formulation.

\section{Filtering of the low Mach number equations}

The large-eddy simulation is based on the idea of scale separation. Theoretically, the separation is carried out by the application of a filter, denoted $(\cdot)$, on the Navier-Stokes equations. We restrict our discussion in this paper to a spatial filter. The filter is taken to verify the properties of conservation of constants, $\bar{a}=a$ with $a$ a constant, and of linearity, $\overline{\phi+\psi}=\bar{\phi}+\bar{\psi}$ for any $\phi$ and $\psi$ [31]. Note however that the filter may be inhomogeneous and thus not commute with derivation. In the 
following, this spatial filter will be referred to as the classical filter. The formulation of the filtered low Mach number equations involves two kinds of subgrid terms. The first kind arises from the non-commutation of the filter with the derivative and are related to the variations of filter width, that is from the LES mesh inhomogeneity. They are denoted in the form $C_{\alpha}^{\beta}$. The second kind arises from nonlinearities, that is from the non-commutation between the filtering and the multiplication operator. They are denoted in the form $F_{\alpha}$. Most of them are related to the large variations of the fluid properties, with the notable exception of the subgrid term associated with momentum convection.

\subsection{Classical formulation}

In the Classical formulation, the low Mach number equations are filtered with the classical filter and expressed in terms of classical-filtered variables. The filtered low Mach number equations in the Classical formulation are given by:

- Mass conservation equation

$$
\frac{\partial \bar{\rho}}{\partial t}+\frac{\partial}{\partial x_{j}}\left(\bar{\rho} \bar{U}_{j}+F_{\rho U_{j}}\right)+C_{\rho U_{j}}^{j}=0
$$

- Momentum conservation equation

$$
\begin{aligned}
\frac{\partial}{\partial t}\left(\bar{\rho} \bar{U}_{i}+F_{\rho U_{i}}\right)= & -\frac{\partial}{\partial x_{j}}\left(\bar{\rho} \bar{U}_{j} \bar{U}_{i}+F_{\rho U_{j} U_{i}}\right)-C_{\rho U_{j} U_{i}}^{j}-\frac{\partial \bar{P}}{\partial x_{i}}-C_{P}^{i} \\
& +\frac{\partial}{\partial x_{j}}\left(\check{\Sigma}_{i j}+F_{\Sigma_{i j}}\right)+C_{\Sigma_{i j}}^{j}
\end{aligned}
$$

- Energy conservation equation

$$
\frac{\partial \bar{U}_{j}}{\partial x_{j}}+C_{U_{j}}^{j}=-\frac{\gamma-1}{\gamma P_{0}}\left[\frac{\partial}{\partial x_{j}}\left(\check{Q}_{j}+F_{Q_{j}}\right)+C_{Q_{j}}^{j}\right]-\frac{1}{\gamma P_{0}} \frac{\partial P_{0}}{\partial t}
$$

- Ideal gas law

$$
\bar{T}=\frac{P_{0}}{r}\left(\frac{1}{\bar{\rho}}+F_{1 / \rho}\right),
$$

with the classical filter counterparts of the shear-stress tensor and of the heat flux given by

$$
\begin{gathered}
\check{\Sigma}_{i j}=\mu(\bar{T})\left(\frac{\partial \bar{U}_{i}}{\partial x_{j}}+\frac{\partial \bar{U}_{j}}{\partial x_{i}}\right)-\frac{2}{3} \mu(\bar{T}) \frac{\partial \bar{U}_{k}}{\partial x_{k}} \delta_{i j}, \\
\check{Q}_{j}=-\lambda(\bar{T}) \frac{\partial \bar{T}}{\partial x_{j}},
\end{gathered}
$$

with $\mu$ the dynamic viscosity, $\lambda$ the thermal conductivity and the following subgrid terms: 


$$
\begin{aligned}
C_{\rho U_{j}}^{j} & =\frac{\overline{\partial \rho U_{j}}}{\partial x_{j}}-\frac{\partial \overline{\rho U_{j}}}{\partial x_{j}} \\
C_{\rho U_{j} U_{i}}^{j} & =\frac{\overline{\partial \rho U_{j} U_{i}}}{\partial x_{j}}-\frac{\partial \overline{\rho U_{j} U_{i}}}{\partial x_{j}} \\
C_{P}^{i} & =\frac{\overline{\partial P}}{\partial x_{i}}-\frac{\partial \bar{P}}{\partial x_{i}} \\
C_{\Sigma_{i j}}^{i} & =\frac{\overline{\partial \Sigma_{i j}}}{\partial x_{j}}-\frac{\partial \overline{\Sigma_{i j}}}{\partial x_{j}} \\
C_{U_{j}}^{j} & =\frac{\overline{\partial U_{j}}}{\partial x_{j}}-\frac{\partial \bar{U}_{j}}{\partial x_{j}}
\end{aligned}
$$

$$
\begin{aligned}
C_{Q_{j}}^{j} & =\frac{\overline{\partial Q_{j}}}{\partial x_{j}}-\frac{\partial \overline{Q_{j}}}{\partial x_{j}} \\
F_{\rho U_{j}} & =\overline{\rho U_{j}}-\bar{\rho} \bar{U}_{j} \\
F_{\rho U_{i}} & =\overline{\rho U_{i}}-\bar{\rho} \bar{U}_{i} \\
F_{\rho U_{j} U_{i}} & =\overline{\rho U_{j} U_{i}}-\bar{\rho}_{j} \bar{U}_{i} \\
F_{\Sigma_{i j}} & =\overline{\Sigma_{i j}}-\check{\Sigma}_{i j} \\
F_{Q_{j}} & =\overline{Q_{j}}-\check{Q}_{j} \\
F_{1 / \rho} & =\frac{\overline{1}}{\rho}-\frac{1}{\bar{\rho}}
\end{aligned}
$$

In addition, it is useful to express the transport equation of the resolved kinetic energy $\bar{\rho} \underline{E}=\frac{1}{2} \bar{\rho} \bar{U}_{i} \bar{U}_{i}$. Indeed, the total energy conservation is not explicitly stated in the system of equations (9)-(12) but implied by the momentum conservation equation (10) and the energy conservation equation (11). The resolved kinetic energy transport equation is the part of total energy conservation related to the momentum conservation. It is obtained from equation (10) multiplied by $\bar{U}_{i}$ and equation (9),

$$
\begin{aligned}
\frac{\partial \bar{\rho} \underline{\underline{E}}}{\partial t}- & \frac{\partial \underline{E} F_{\rho U_{j}}}{\partial x_{j}}+F_{\rho U_{j}} \frac{\partial \underline{E}}{\partial x_{j}}-\underline{E} C_{\rho U_{j}}^{j}+\frac{\partial \bar{U}_{i} F_{\rho U_{i}}}{\partial t}-F_{\rho U_{i}} \frac{\partial \bar{U}_{i}}{\partial t} \\
= & -\frac{\partial}{\partial x_{j}}\left(\bar{\rho} \bar{U}_{j} \underline{E}+\bar{U}_{i} F_{\rho U_{j} U_{i}}\right)+F_{\rho U_{j} U_{i}} \frac{\partial \bar{U}_{i}}{\partial x_{j}}-\bar{U}_{i} C_{\rho U_{j} U_{i}}^{j}-\frac{\partial \bar{U}_{i} \bar{P}}{\partial x_{i}}+\bar{P} \frac{\partial \bar{U}_{i}}{\partial x_{i}}-\bar{U}_{i} C_{P}^{i} \\
& +\frac{\partial}{\partial x_{j}}\left(\bar{U}_{i} \check{\Sigma}_{i j}+\bar{U}_{i} F_{\Sigma_{i j}}\right)-\left(\check{\Sigma}_{i j}+F_{\Sigma_{i j}}\right) \frac{\partial \bar{U}_{i}}{\partial x_{j}}+\bar{U}_{i} C_{\Sigma_{i j}}^{j} .
\end{aligned}
$$

This equation gives the contribution of the subgrid terms of the filtered momentum conservation equation to the balance of the resolved kinetic energy. It will be used to assess the magnitude of their energetic contribution. The contribution of the subgrid terms with regard to total energy is given by the subgrid terms of the resolved kinetic energy transport equation (27) and the energy conservation equation (11).

\subsection{Favre formulation}

The use of the Favre filter $(\widetilde{*})$ is common when working with variable density flows. It is a variable change in which filtered variables are weighted by density. For any variable $\phi$, the Favre-filtered variable $\widetilde{\phi}$ is defined as $\widetilde{\phi}=\overline{\rho \phi} / \bar{\rho}$. With the Favre filter, we avoid the subgrid terms associated with the nonlinearities of the form $\rho \phi$ in the convective term of the mass conservation equation, the time derivative term of the momentum conservation equation and the ideal gas law. On the other hand, a subgrid term is added when a variable $\phi$ appears without the density, as the velocity in the energy conservation equation. The subgrid terms associated with the nonlinearities of the shear-stress tensor and of the conductive heat flux are modified by the use of the Favre filter, but it is not obvious to what extent this affects the behaviour and importance of the subgrid terms. Finally, the 
subgrid terms associated with the non-commutation of the filter with the derivative are not modified by the use of the Favre filter.

In the Favre formulation, the low Mach number equations are filtered with the classical filter and expressed in terms of Favre-filtered variables. The filtered low Mach number equations in the Favre formulation are given by:

- Mass conservation equation

$$
\frac{\partial \bar{\rho}}{\partial t}+\frac{\partial \bar{\rho} \widetilde{U_{j}}}{\partial x_{j}}+C_{\rho U_{j}}^{j}=0,
$$

- Momentum conservation equation

$$
\begin{aligned}
\frac{\partial \bar{\rho} \widetilde{U}_{i}}{\partial t}= & -\frac{\partial}{\partial x_{j}}\left(\bar{\rho} \widetilde{U}_{j} \widetilde{U}_{i}+\bar{\rho} G_{U_{j} U_{i}}\right)-C_{\rho U_{j} U_{i}}^{j}-\frac{\partial \bar{P}}{\partial x_{i}}-C_{P}^{i} \\
& +\frac{\partial}{\partial x_{j}}\left(\widehat{\Sigma}_{i j}+G_{\Sigma_{i j}}\right)+C_{\Sigma_{i j}}^{j},
\end{aligned}
$$

- Energy conservation equation

$$
\frac{\partial}{\partial x_{j}}\left(\widetilde{U}_{j}+\bar{\rho} G_{U_{j} / \rho}\right)+C_{U_{j}}^{j}=-\frac{\gamma-1}{\gamma P_{0}}\left[\frac{\partial}{\partial x_{j}}\left(\widehat{Q}_{j}+G_{Q_{j}}\right)+C_{Q_{j}}^{j}\right]-\frac{1}{\gamma P_{0}} \frac{\partial P_{0}}{\partial t},
$$

- Ideal gas law

$$
\widetilde{T}=\frac{P_{0}}{\bar{\rho} r}
$$

with the Favre filter counterparts of the shear-stress tensor and of the heat flux given by

$$
\begin{gathered}
\widehat{\Sigma}_{i j}=\mu(\widetilde{T})\left(\frac{\partial \widetilde{U}_{i}}{\partial x_{j}}+\frac{\partial \widetilde{U}_{j}}{\partial x_{i}}\right)-\frac{2}{3} \mu(\widetilde{T}) \frac{\partial \widetilde{U}_{k}}{\partial x_{k}} \delta_{i j}, \\
\widehat{Q}_{j}=-\lambda(\widetilde{T}) \frac{\partial \widetilde{T}}{\partial x_{j}},
\end{gathered}
$$

and the following subgrid terms specific to the Favre formulation:

$$
\begin{aligned}
G_{U_{j} U_{i}} & =\widetilde{U_{j} U_{i}}-\widetilde{U}_{j} \widetilde{U}_{i} \\
G_{\Sigma_{i j}} & =\overline{\Sigma_{i j}}-\widehat{\Sigma}_{i j}
\end{aligned}
$$

$$
\begin{aligned}
G_{U_{j} / \rho} & =\widetilde{U_{j} / \rho}-\widetilde{U}_{j} / \bar{\rho} \\
G_{Q_{j}} & =\overline{Q_{j}}-\widehat{Q}_{j}
\end{aligned}
$$

The subgrid terms $F_{\rho U_{j}}$ and $G_{U_{j} / \rho}$ are closely related,

$$
\frac{F_{\rho U_{j}}}{\bar{\rho}}=-\bar{\rho} G_{U_{j} / \rho}
$$

These two subgrid terms express explicitly the correlation between density and velocity. 
The resolved kinetic energy transport equation is obtained from equation (29) multiplied by $\widetilde{U}_{i}$ and equation (28),

$$
\begin{aligned}
\frac{\partial \bar{\rho} \underset{\sim}{E}}{\partial t}-\underset{\sim}{E} C_{\rho U_{j}}^{j} \\
=-\frac{\partial}{\partial x_{j}}\left(\bar{\rho} \widetilde{U}_{j} \underset{\sim}{E}+\bar{\rho} \widetilde{U}_{i} G_{U_{j} U_{i}}\right)+\bar{\rho} G_{U_{j} U_{i}} \frac{\partial \widetilde{U}_{i}}{\partial x_{j}}-\widetilde{U}_{i} C_{\rho U_{j} U_{i}}^{j}-\frac{\partial \widetilde{U_{i}} \bar{P}}{\partial x_{i}}+\bar{P} \frac{\partial \widetilde{U}_{i}}{\partial x_{j}}-\widetilde{U}_{i} C_{P}^{i} \\
\quad+\frac{\partial}{\partial x_{j}}\left(\widetilde{U}_{i} \widehat{\Sigma}_{i j}+\widetilde{U}_{i} G_{\Sigma_{i j}}\right)-\left(\widehat{\Sigma}_{i j}+G_{\Sigma_{i j}}\right) \frac{\partial \widetilde{U}_{i}}{\partial x_{j}}+\widetilde{U}_{i} C_{\Sigma_{i j}}^{j} .
\end{aligned}
$$

Compared to the Classical formulation, the Favre formulation is a priori more suited to the momentum conservation equation, the mass conservation equation and the ideal gas law but is less appropriate for the energy conservation equation.

\subsection{Velocity formulation}

The Velocity formulation is based on the velocity filtering approach suggested by Sidharth and Candler [35], Sidharth et al. [36]. The momentum conservation equation in the low Mach number equations is rewritten before filtering as the velocity transport equation,

$$
\frac{\partial U_{i}}{\partial t}=-\frac{\partial U_{j} U_{i}}{\partial x_{j}}+U_{i} \frac{\partial U_{j}}{\partial x_{j}}-\frac{1}{\rho} \frac{\partial P}{\partial x_{i}}+\frac{1}{\rho} \frac{\partial \Sigma_{i j}}{\partial x_{j}} .
$$

The equations are then filtered with the classical filter and expressed in terms of classical-filtered variables. The filtered low Mach number equations in the Velocity formulation are given by the mass conservation equation (9), the energy conservation equation (11), the ideal gas law (12) and the velocity transport equation:

$$
\begin{aligned}
\bar{\rho} \frac{\partial \bar{U}_{i}}{\partial t}= & -\bar{\rho} \frac{\partial}{\partial x_{j}}\left(\bar{U}_{j} \bar{U}_{i}+F_{U_{j} U_{i}}\right)-\bar{\rho} C_{U_{j} U_{i}}^{j}+\bar{\rho} \bar{U}_{i} \frac{\partial \bar{U}_{j}}{\partial x_{j}}+\bar{\rho} F_{U_{i} \partial_{j} U_{j}}+\bar{\rho} \bar{U}_{i} C_{U_{j}}^{j} \\
& -\frac{\partial \bar{P}}{\partial x_{i}}-C_{P}^{i}-\bar{\rho} F_{\partial_{i} P / \rho}+\frac{\partial}{\partial x_{j}}\left(\check{\Sigma}_{i j}+F_{\Sigma_{i j}}\right)+C_{\Sigma_{i j}}^{j}+\bar{\rho} F_{\partial_{j} \Sigma_{i j} / \rho},
\end{aligned}
$$

with the following subgrid terms:

$$
\begin{aligned}
C_{U_{j} U_{i}}^{j} & =\frac{\overline{\partial U_{j} U_{i}}}{\partial x_{j}}-\frac{\partial \overline{U_{j} U_{i}}}{\partial x_{j}} \\
F_{U_{j} U_{i}} & =\overline{U_{j} U_{i}}-\bar{U}_{j} \bar{U}_{i} \\
F_{U_{i} \partial_{j} U_{j}} & =U_{i} \frac{\partial U_{j}}{\partial x_{j}}-\bar{U}_{i} \frac{\overline{\partial U_{j}}}{\partial x_{j}}
\end{aligned}
$$

$$
\begin{aligned}
F_{\partial_{i} P / \rho} & =\frac{\overline{1} \frac{\partial P}{\partial x_{i}}}{\rho}-\frac{1}{\bar{\rho}} \frac{\overline{\partial P}}{\partial x_{i}} \\
F_{\partial_{j} \Sigma_{i j} / \rho} & =\frac{\overline{1} \frac{\partial \Sigma_{i j}}{\partial x_{j}}}{\rho}-\frac{1}{\bar{\rho} \frac{\overline{\partial \Sigma_{i j}}}{\partial x_{j}}}
\end{aligned}
$$

Upon filtering, the velocity transport equation gives rise to different subgrid terms than the momentum conservation equation. The time derivative of the velocity density product subgrid term $F_{\rho U_{i}}$ does not appear in the Velocity formulation. 
However, there is three additional subgrid terms associated with the divergence of the velocity and the correlation of the density with other terms of the equation.

The resolved kinetic energy transport equation is given by

$$
\begin{aligned}
\bar{\rho} \frac{\partial \underline{E}}{\partial t}= & -\bar{\rho} \frac{\partial}{\partial x_{j}}\left(\bar{U}_{j} \underline{E}+F_{U_{j} U_{i}} \bar{U}_{i}\right)+\bar{\rho} F_{U_{j} U_{i}} \frac{\partial \bar{U}_{i}}{\partial x_{j}}-\bar{\rho} \bar{U}_{i} C_{U_{j} U_{i}}^{j} \\
& +\bar{\rho} \underline{\underline{E}} \frac{\partial \bar{U}_{j}}{\partial x_{j}}+\bar{\rho} \bar{U}_{i} F_{U_{i} \partial_{j} U_{j}}+2 \bar{\rho} \underline{E} C_{U_{j}}^{j} \\
& -\frac{\partial \bar{P} \bar{U}_{i}}{\partial x_{i}}+\bar{P} \frac{\partial \bar{U}_{i}}{\partial x_{i}}-\bar{U}_{i} C_{P}^{i}-\bar{\rho}_{i} F_{\partial_{i} P / \rho} \\
& +\frac{\partial}{\partial x_{j}}\left(\check{\Sigma}_{i j} \bar{U}_{i}+F_{\Sigma_{i j}} \bar{U}_{i}\right)-\left(\check{\Sigma}_{i j}+F_{\Sigma_{i j}}\right) \frac{\partial \bar{U}_{i}}{\partial x_{j}}+\bar{U}_{i} C_{\Sigma_{i j}}^{j}+\bar{\rho}_{i} \bar{U}_{\partial_{j} \Sigma_{i j} / \rho} .
\end{aligned}
$$

This equation is obtained from equation (41) multiplied by $\bar{U}_{i}$ and equation (9).

\section{Numerical study configuration}

\subsection{Channel flow configuration}

We consider a fully developed three-dimensional anisothermal channel flow, as shown in figure 1. This geometry is one of the simpler that reproduces the distinctive features of low Mach number strongly anisothermal turbulent flows. It is therefore well suited to the study of the subgrid scale specificities of these flows.

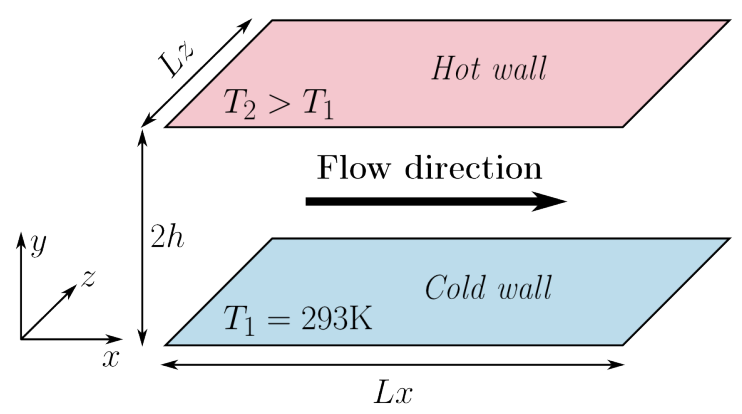

Figure 1 - Biperiodic anisothermal channel flow.

The channel is periodic in both the streamwise $(x)$ and spanwise $(z)$ directions. The temperatures of the two plane channel walls are $T_{2}=586 \mathrm{~K}$ at the hot wall and $T_{1}=293 \mathrm{~K}$ at the cold wall. This creates the temperature gradient in the wall-normal direction $(y)$. Two mean friction Reynolds number are considered, $R e_{\tau}$ $=180$ and $R e_{\tau}=395$, where $R e_{\tau}$ is defined as the average of the friction Reynolds numbers $R e_{\tau, \omega}$ calculated at the hot and cold wall,

$$
R e_{\tau, \omega}=\frac{U_{\tau} h}{\nu_{\omega}}
$$


with $U_{\tau}=\nu_{\omega}\left(\partial_{y} \bar{U}_{x}\right)_{\omega}^{0.5}$ the friction velocity and $\nu_{\omega}$ the wall kinematic viscosity. We show in figure 2 an isotherm and the velocity magnitude on a plane for a given timestep at $R e_{\tau}=180$.

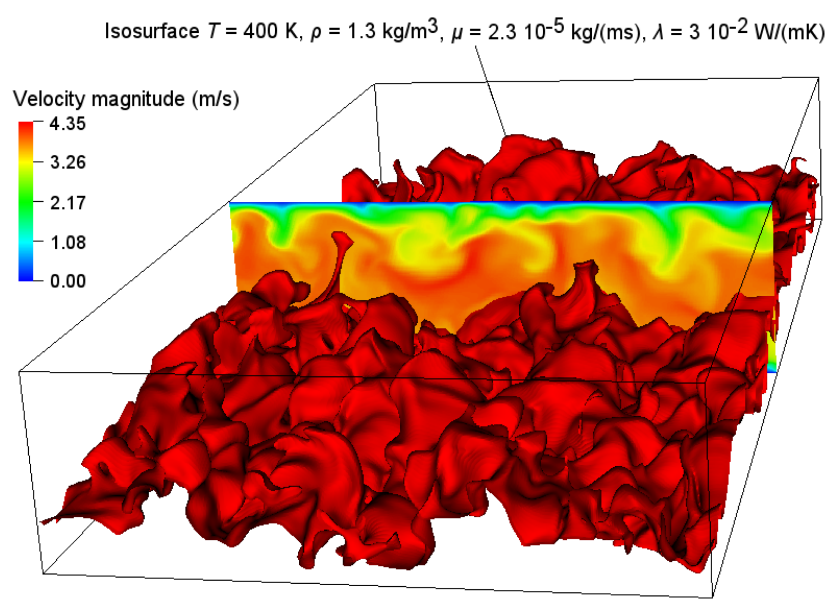

Figure 2 - Surface of the $400 \mathrm{~K}$ isotherm and velocity magnitude in a plane normal to the flow direction $\left(R e_{\tau}=180\right)$.

\subsection{Numerical settings}

To provide the data required to compute the subgrid terms of the low Mach number equations, direct numerical simulations of the fully developed channel flow described in 4.1 are carried for the two selected friction Reynolds number (180 and 395). At $R e_{\tau}=180$, the domain size is $4 \pi h \times 2 h \times 2 \pi h$, and the mesh used contains $384 \times 266 \times 384$ grid points. At $R e_{\tau}=395$, the domain size is $4 \pi h \times 2 h \times 4 / 3 \pi h$ and the mesh used contains $768 \times 512 \times 512$ grid points. In both cases, the mesh is regular in both homogeneous directions and follow a hyperbolic tangent law in the wall-normal coordinate direction. The wall-normal grid coordinates are given by

$$
y_{k}=L_{y}\left(1+\frac{1}{a} \tanh \left[\left(\frac{k-1}{N_{y}-1}-1\right) \tanh ^{-1}(a)\right]\right) \text {, }
$$

with $a$ the mesh dilatation parameter and $N_{y}$ the number of grid points in the wall-normal direction. The cell sizes in wall units are $\Delta_{x}^{+}=8.5, \Delta_{y}^{+}=0.13-4.2$ and $\Delta_{z}^{+}=4.2$ at $R e_{\tau}=180 ; \Delta_{x}^{+}=9.1, \Delta_{y}^{+}=0.25-4.1$ and $\Delta_{z}^{+}=4.7$ at $R e_{\tau}=$ 395. The two meshes have the same level of refinement. The small differences are due to the constraints of the numerical method (multigrid solver) and parallelism. A finite volume method is used with a third-order Runge-Kutta time scheme and a fourth-order centred momentum convection scheme. This is performed using the TrioCFD software [9]. The numerical set-up is validated through a mesh convergence study and by comparison of our results in the incompressible case to the reference data of Moser et al. [27] and Vreman and Kuerten [41]. These numerical results have been validated against experimental data [13, 17, 21, 3]. We provide in figure 3 a comparison of the spatial turbulence kinetic energy terms computed by our 


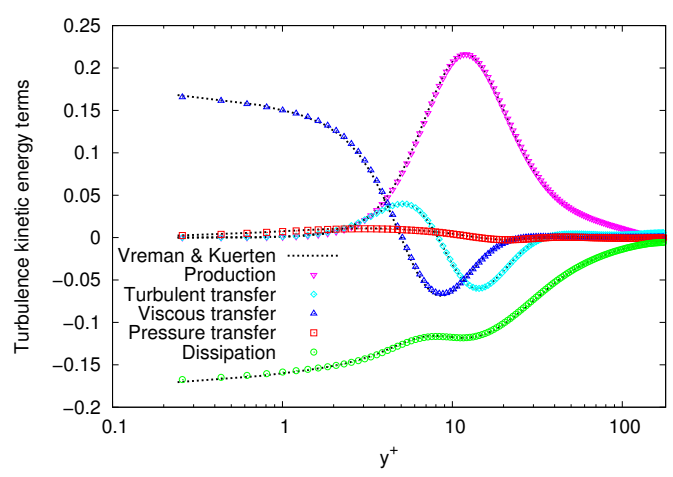

Figure 3 - Validation of the spatial turbulence kinetic energy terms.

numerical procedure to the reference data of Vreman and Kuerten [41] at $R e_{\tau}=$ 180. Similarly, the results have been compared to the reference data of Moser et al. [27] at $R e_{\tau}=395$ (not shown here). This validates our numerical method at the incompressible limit. In the anisothermal configuration, the same code has been validated against experimental data for a similar friction Reynolds number and temperature gradient by Bellec et al. [6].

\subsection{Filtering process}

To compute the subgrid terms a priori, we filter explicitly the DNS flow field. At $R e_{\tau}=180$, three filters of varying width are investigated, from now on called "filter A", "filter B" and "filter C". The three filters are three-dimensional box filter, also known as top-hat filter, of uniform width in both homogeneous directions and nonuniform width in the wall-normal direction. The filter sizes in wall units and the number of grid points corresponding to the filters are,

- filter A: $\bar{\Delta}_{x}^{+}=43, \bar{\Delta}_{y}^{+}=0.8-21, \bar{\Delta}_{z}^{+}=21$ and $77 \times 53 \times 77$ grid points;

- filter B: $\bar{\Delta}_{x}^{+}=68, \bar{\Delta}_{y}^{+}=0.5-25, \bar{\Delta}_{z}^{+}=34$ and $48 \times 50 \times 48$ grid points;

- filter C: $\bar{\Delta}_{x}^{+}=91, \bar{\Delta}_{y}^{+}=0.13-43, \bar{\Delta}_{z}^{+}=45$ and $36 \times 40 \times 36$ grid points.

At $R e_{\tau}=395$, only filter A is examined. The filter sizes in wall units are $\bar{\Delta}_{x}^{+}=47$, $\bar{\Delta}_{y}^{+}=1.5-21$ and $\bar{\Delta}_{z}^{+}=24$. This corresponds to a mesh with $154 \times 102 \times 102$ grid points.

The filter A corresponds to an average over five DNS cells in the three directions. It is computed using the following discrete approximation of the box filter:

$$
\bar{\phi}\left(x_{i}, y_{k}, z_{j}, t\right)=\frac{1}{25\left(y_{k^{\prime}+3}-y_{k^{\prime}-2}\right)} \sum_{i^{\prime}=i-2}^{i+2} \sum_{k^{\prime}=k-2}^{k+2} \sum_{j^{\prime}=j-2}^{j+2} \phi\left(x_{i^{\prime}}, y_{k^{\prime}}, z_{j^{\prime}}, t\right)\left(y_{k^{\prime}+1}-y_{k^{\prime}}\right) .
$$

The variations of the filter width in the wall-normal direction follow those of the DNS mesh. 
The filters B and C are constructed to follow a hyperbolic tangent law (49) in the wall-normal direction. We cannot use the same method as for filter A to compute the filters $\mathrm{B}$ and $\mathrm{C}$ because the filter width is a non-integer multiple of the DNS cell size. In order to carry out the filtering with an arbitrary filter length, the DNS data are first interpolated using a cubic spline. Then, the top-hat filter is computed from the interpolated values without mesh restrictions. The spline interpolation adds an additional filtering to the box filter. However, this additional filter can be neglected given the resolution of the direct numerical simulation. The interpolation and filtering are not computed in three dimensions but sequentially in the three spatial directions. This is possible because the box filter is separable, that is, can be expressed as the product of three one-dimensional filters. Using the filter width of filter A, the method given similar results to equation (50).

The filtered quantities are not computed very close the domain boundary, where not enough points are available to apply the filter. This is justified by the fact that in practice, the large-eddy simulation of the channel would be carried out with DNS precision very close to the wall.

\section{Results and discussion}

We assess the importance of the subgrid terms in the Classical formulation, the Favre formulation and the Velocity formulation. This provides valuable data to determine which terms can be neglected and which terms should be modelled. The strength of the subgrid terms are investigated according to their quadratic mean, or root mean square, compared to the non-subgrid terms. Numerically, the quadratic mean is computed by an average on the two homogeneous directions and on 100 non-consecutive time steps that cover all thermodynamic configurations of the flow. The results are converged as the mean computed on 50 time steps is identical to the mean computed on 100 time steps. Since the flow is homogeneous in the streamwise and spanwise directions, the analysis is carried out as a function of the wall-normal coordinate $y$, scaled by the height of the channel and in the classical wall scaling

$$
y^{+}=R e_{\tau} \frac{y}{h}=\frac{y U_{\tau}}{\nu_{\omega}} .
$$

The subgrid terms are first investigated with the filter A. Then, the effect of the filter width on the results is examined.

\subsection{Magnitude of the subgrid terms}

In this section, the magnitude of subgrid terms is assessed at $R e_{\tau}=180$ and $R e_{\tau}=395$ with the filter A. The results at $R e_{\tau}=180$ and $R e_{\tau}=395$ are identical with regard to the classification of the subgrid terms. We thus only provide the results with the filter $\mathrm{A}$ at $R e_{\tau}=180$, for the mass conservation equation (figure 4 ), the streamwise momentum conservation equation or the streamwise velocity transport equation (figure 5), the spanwise momentum conservation equation or the 
spanwise velocity transport equation (figure 6), the wall-normal momentum conservation equation or the wall-normal velocity transport equation (figure 7 ), the resolved kinetic energy transport equation (figure 8), the energy conservation equation (figure 9), and the ideal gas law (figure 10). In each case, the results are given in the entire channel with a linear abscissa and at the cold side with a logarithmic abscissa, to emphasise the near-wall region. Only the cold side is shown with the logarithmic abscissa because a large-eddy simulation of the channel would be less resolved at the cold side than at the hot side.

Indeed, given the dependence of density, viscosity and conductivity on temperature, the temperature gradient generates an asymmetry between the hot and cold sides of the channel. The dynamic viscosity and the friction velocity are higher at the hot wall and the friction Reynolds number is larger at the cold side. All terms of the mass conservation equation, momentum conservation equation, resolved kinetic energy transport equation and energy conservation equation have a larger amplitude at the cold side than at the hot side. In addition, the local maxima of the profiles of most subgrid terms are closer to the wall at the cold side than at the hot side. This asymmetry can in a large part be explained by the local variations of the mean fluid properties [39]. The profiles are also subject to a low Reynolds number effect [16]. Since the three filters used are symmetric with respect to the centre of the channel, the cold side is less resolved in wall units than the hot side. This contributes to the fact that the subgrid terms have a larger amplitude at the cold side than at the hot side in the mass conservation equation, momentum conservation equation, resolved kinetic energy transport equation and energy conservation equation.

\subsubsection{Mass conservation equation}

The mass conservation equation (figure 4) gives a relationship between the time derivative of density and the divergence of the mass flux. With the classical filter (Classical or Velocity formulation), the subgrid term associated with the densityvelocity correlation $\partial_{j} F_{\rho U_{j}}$ is very small at the centre of the channel and remains one order of magnitude smaller than the non-subgrid terms near the wall, where it is the most significant. The filter-derivative non-commutation subgrid term $C_{\rho U_{j}}^{j}$ has a smaller amplitude in most part of the channel but has the same order of magnitude at the wall. With the Favre filter, it is the only subgrid term in the mass conservation equation.

Since the statistical average of the time derivative of density is zero, the divergence of the resolved mass flux $\partial_{j} \bar{\rho} \overline{U_{j}}$ is in balance with the subgrid terms. With the classical filter, the statistical average of the density-velocity correlation subgrid term has the same order of magnitude as the divergence of the resolved mass flux, while the filter-derivative non-commutation subgrid term is negligible. The modelling of the subgrid term $\partial_{j} \bar{\rho} \bar{U}_{j}$ is necessary to take this behaviour into account in a large-eddy simulation. Therefore, we consider that with the classical filter, the modelling of the density-velocity correlation subgrid term $\partial_{j} F_{\rho U_{j}}$ is more important than the modelling of the filter-derivative non-commutation subgrid term $C_{\rho U_{j}}^{j}$. 

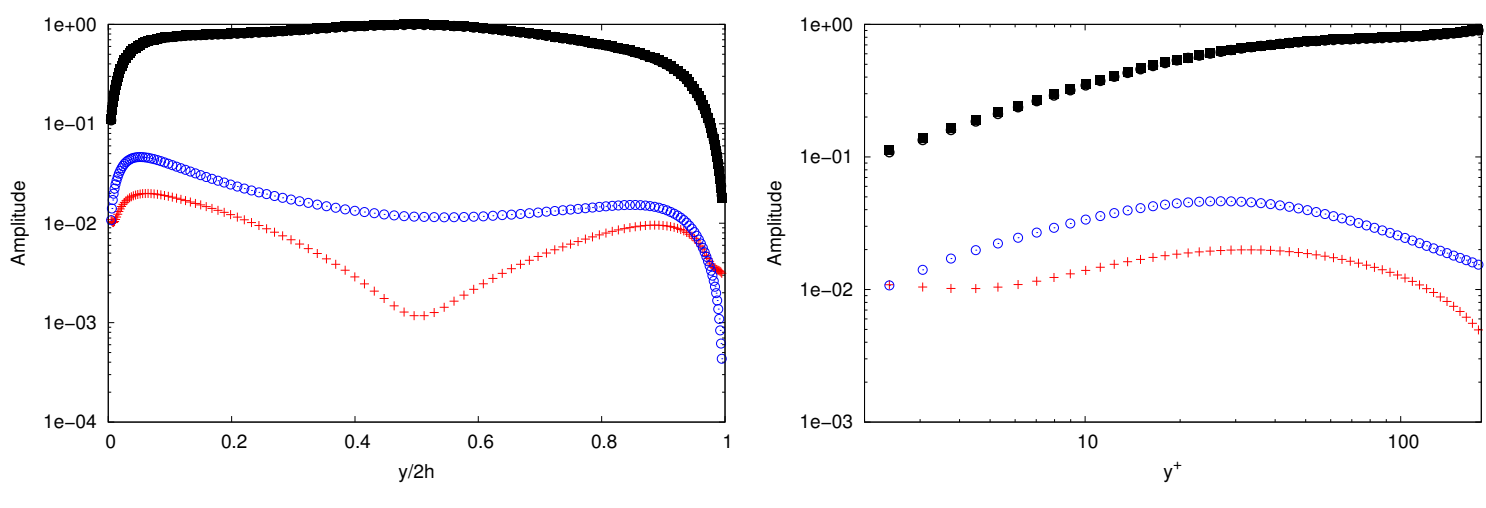

(a) With the classical filter.
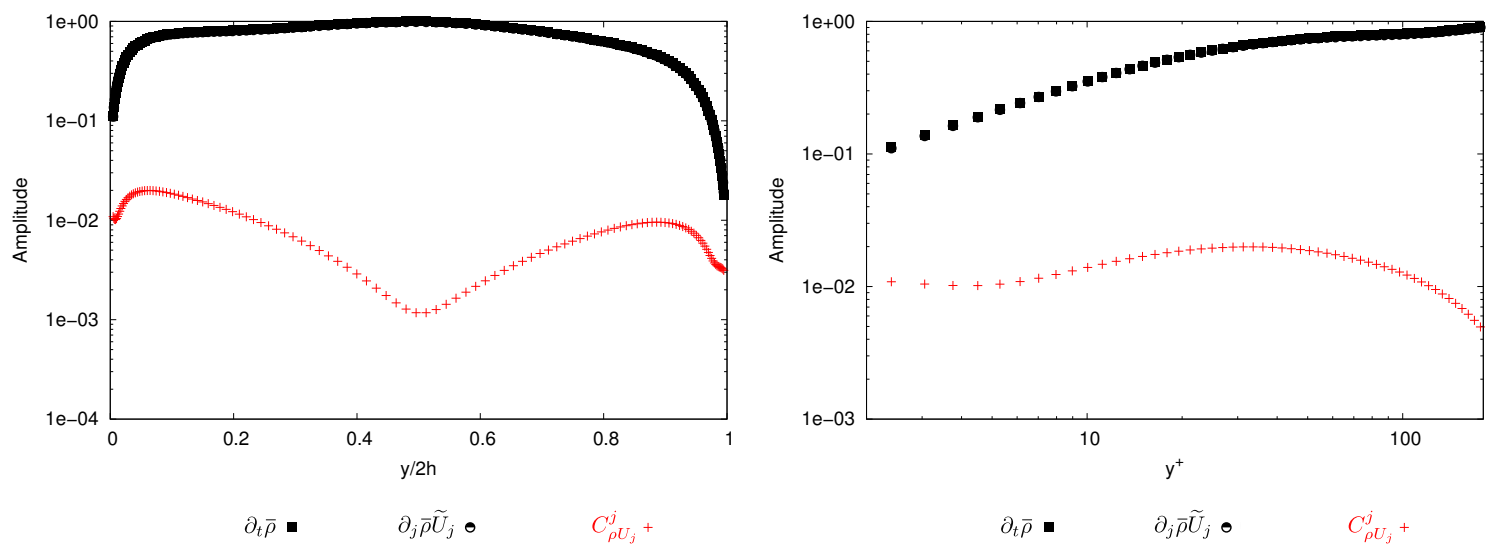

(b) With the Favre filter.

Figure 4 - Root mean square of the terms of the mass conservation equation as a function of the wall-normal coordinate in the Classical and Velocity formulations (a) and in the Favre formulation (b) at $R e_{\tau}=180$ with the filter A. The amplitude is scaled by the maximum value in the domain in the three formulations.

\subsubsection{Momentum conservation equation}

To study the subgrid terms related to the momentum conservation, we investigate the subgrid terms as they appear in the streamwise, spanwise and wall-normal momentum conservation equations (figures 5, 6 and 7 respectively), or velocity transport equation in the case of the Velocity formulation, and the energetic contribution of the subgrid terms from the resolved kinetic energy transport equation (figure 9). In either case, the time derivative term and the convective term predominate at the centre of the channel. Nevertheless, these two terms in a large part cancel each other out. To be considered negligible, a subgrid term should thus at least be negligible compared to the third largest non-subgrid term, namely the pressure term.

Two non-subgrid terms are negligible throughout the entire channel, namely the velocity-dilatation product in the spanwise and wall-normal velocity transport equations and the pressure-dilatation product in the resolved kinetic energy transport 

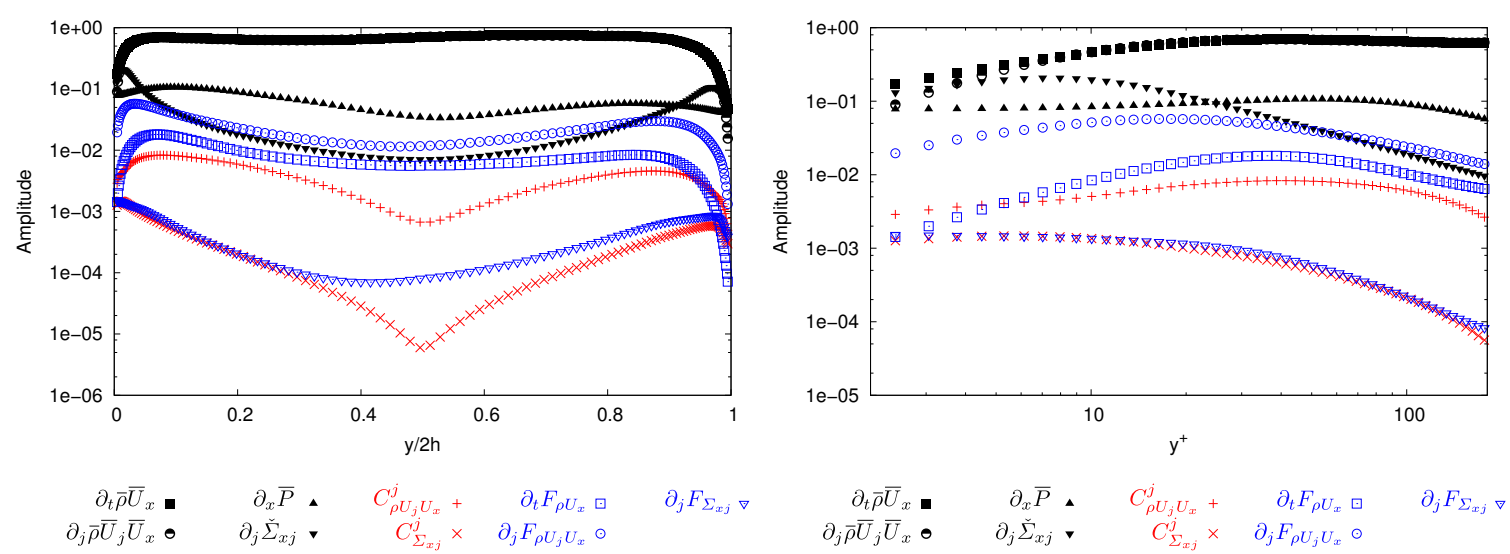

$\partial_{j} \bar{\rho} \bar{U}_{j} \bar{U}_{x} \odot \quad \partial_{j} \check{\Sigma}_{x j} \vee$

$C_{\Sigma_{x j}^{j}}^{j} \times \partial_{j} F_{\rho U_{j} U_{x}} \circ$

(a) In the Classical formulation.
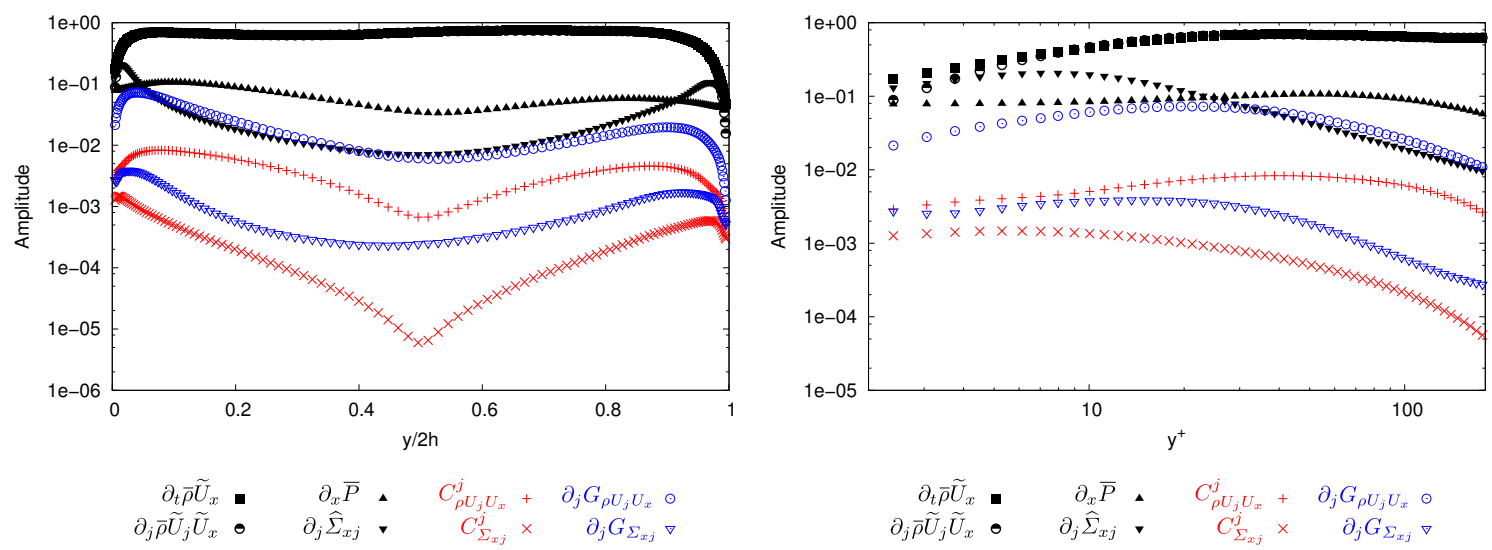

(b) In the Favre formulation.
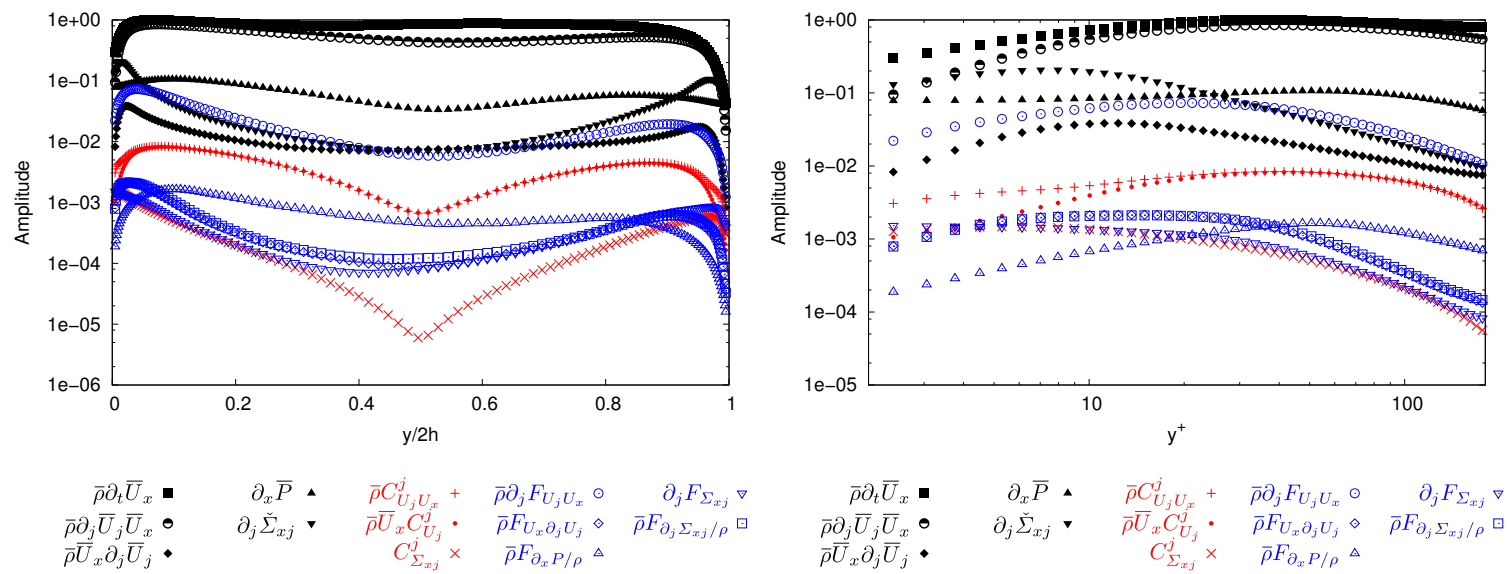

(c) In the Velocity formulation.

Figure 5 - Root mean square of the terms of the streamwise momentum conservation equation as a function of the wall-normal coordinate in the Classical formulation (a), in the Favre formulation (b) and of the terms of the streamwise velocity transport equation in the Velocity formulation (c) at $R e_{\tau}=180$ with the filter A. The amplitude is scaled by the maximum value in the domain in the three formulations. 

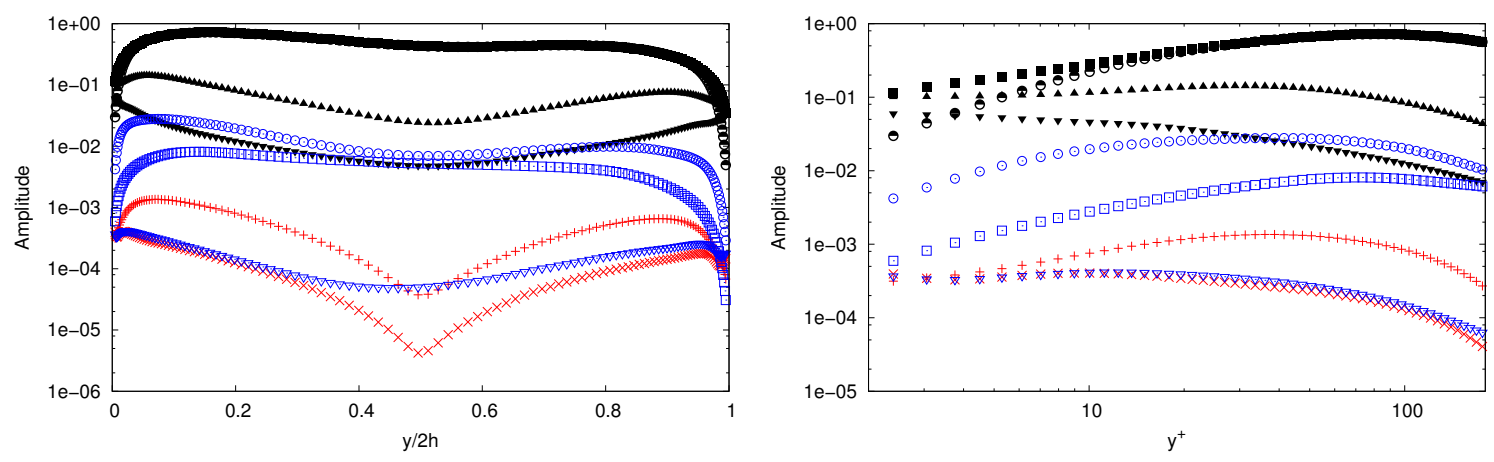

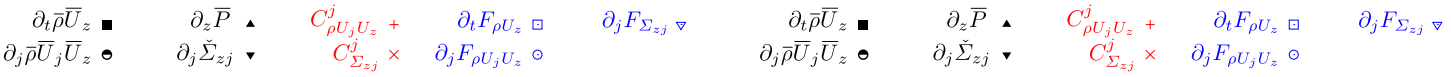

(a) In the Classical formulation.
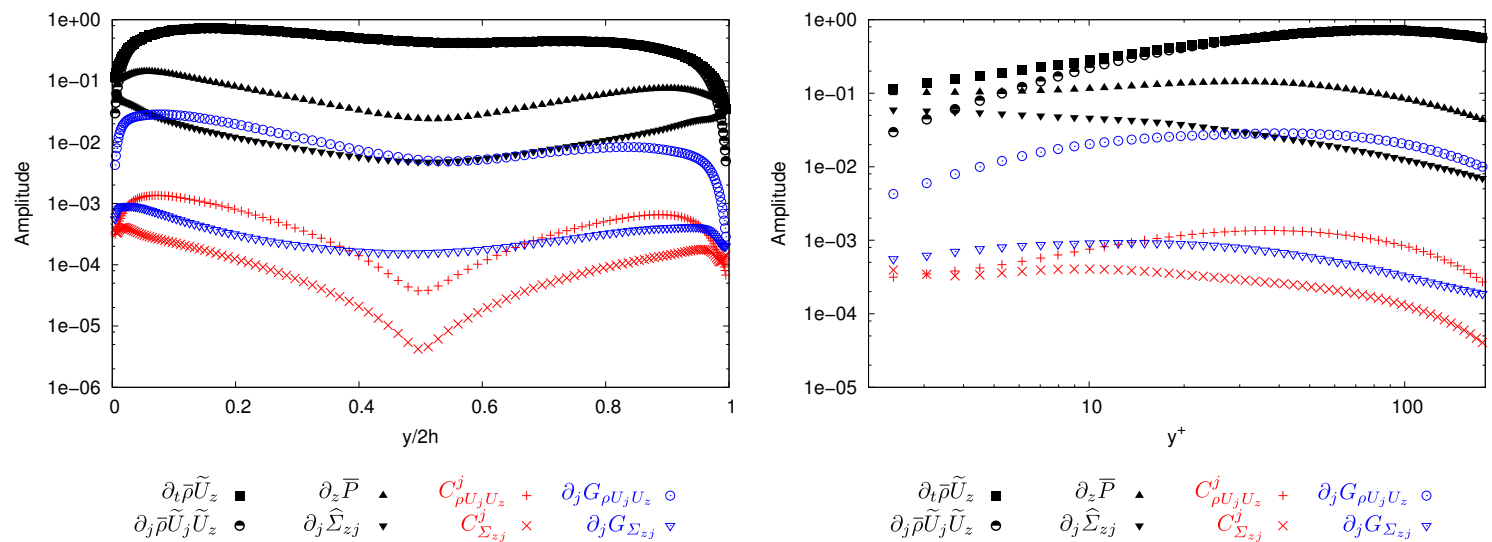

(b) In the Favre formulation.
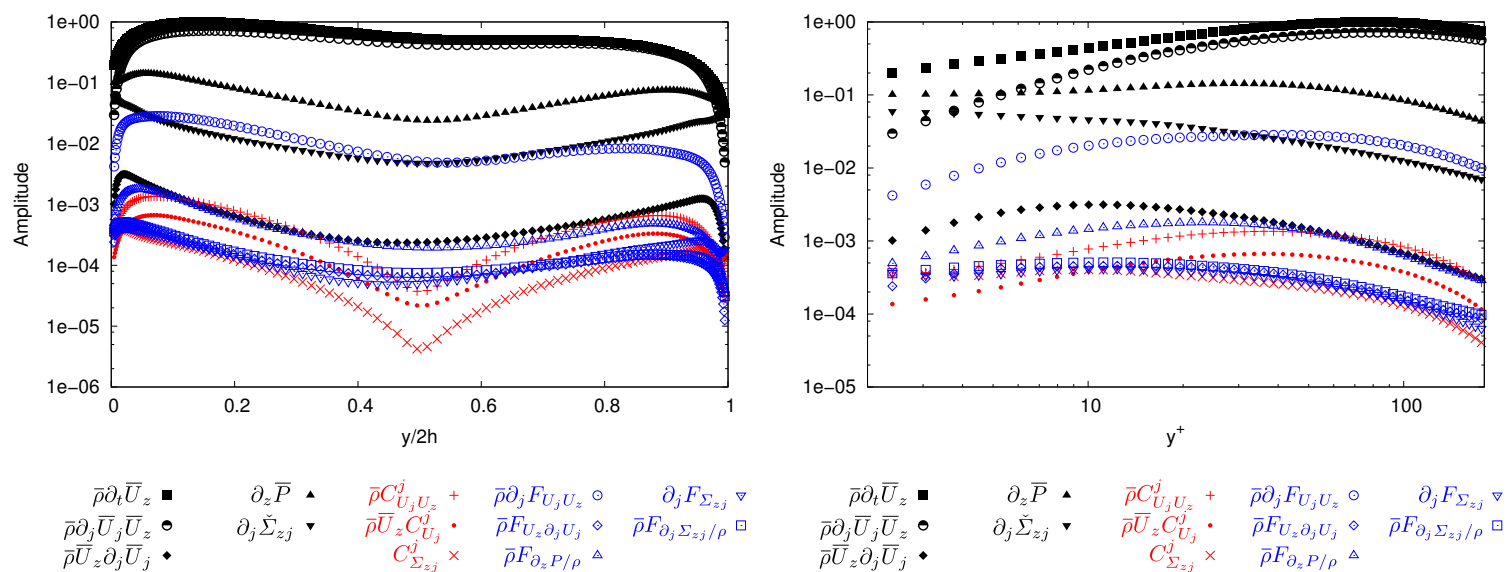

(c) In the Velocity formulation.

Figure 6 - Root mean square of the terms of the spanwise momentum conservation equation as a function of the wall-normal coordinate in the Classical formulation (a), in the Favre formulation (b) and of the terms of the spanwise velocity transport equation in the Velocity formulation (c) at $R e_{\tau}=180$ with the filter A. The amplitude is scaled by the maximum value in the domain in the three formulations. 

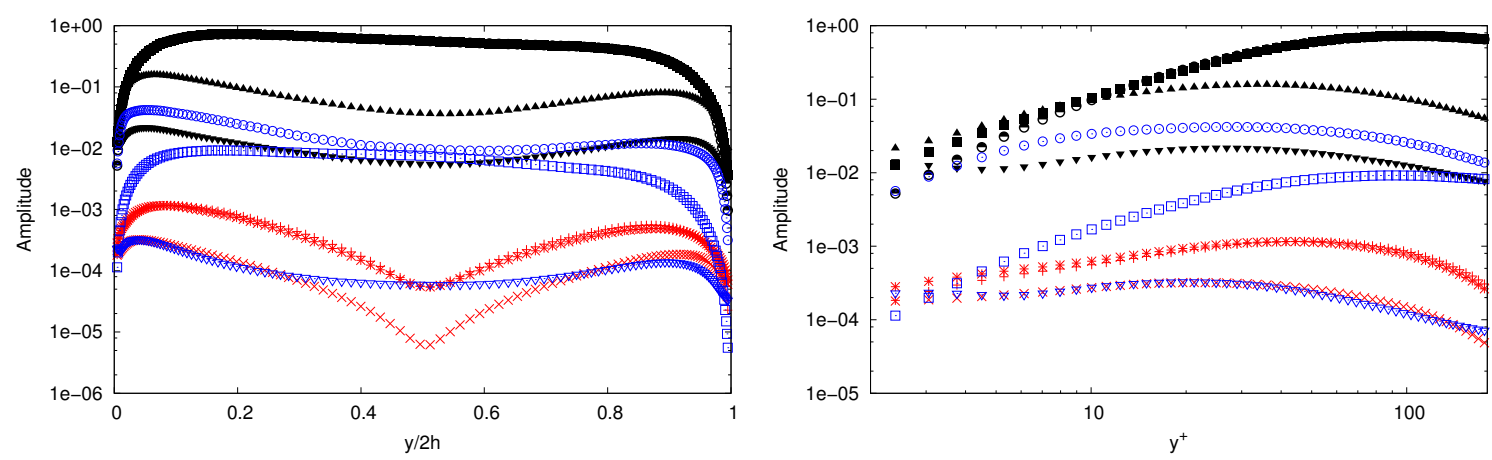

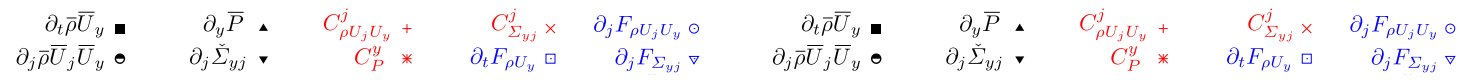

(a) In the Classical formulation.
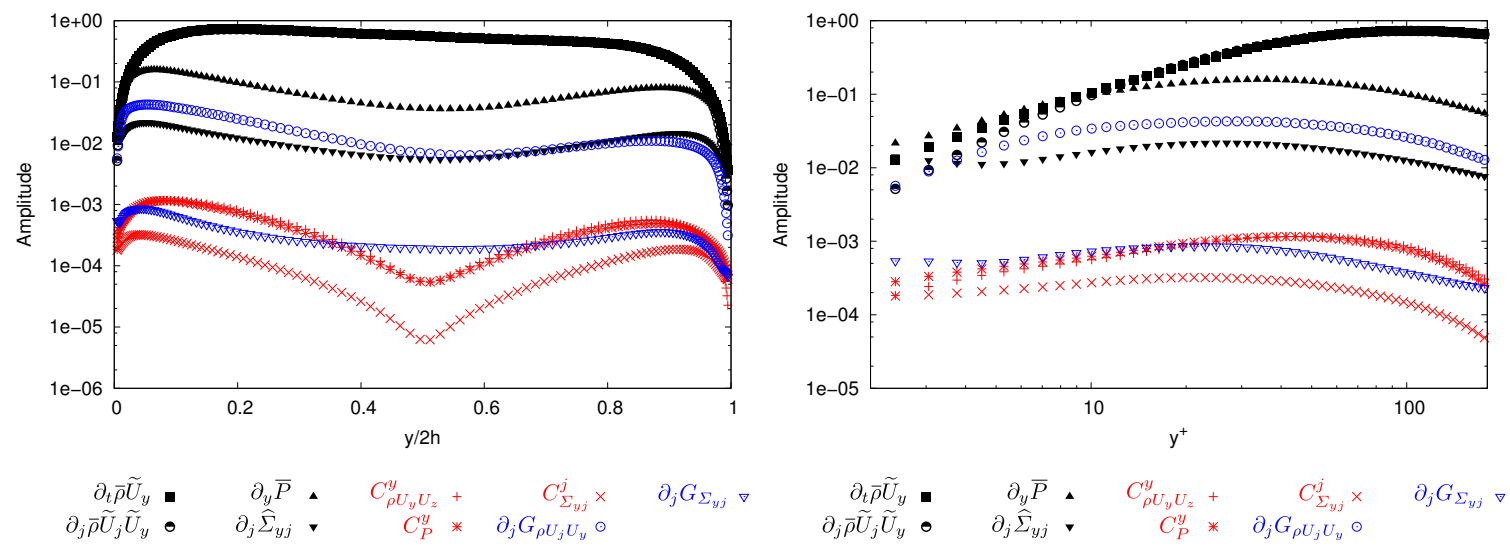

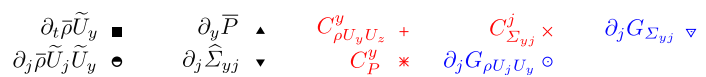

$\partial_{j} \widetilde{\rho}_{j} \widetilde{U}_{y} \odot \quad \partial_{j} \widetilde{\Sigma}_{y j} \cdot \quad C_{P}^{y} * \partial_{j} G_{\rho U_{j} U_{y}} \circ$

(b) In the Favre formulation.
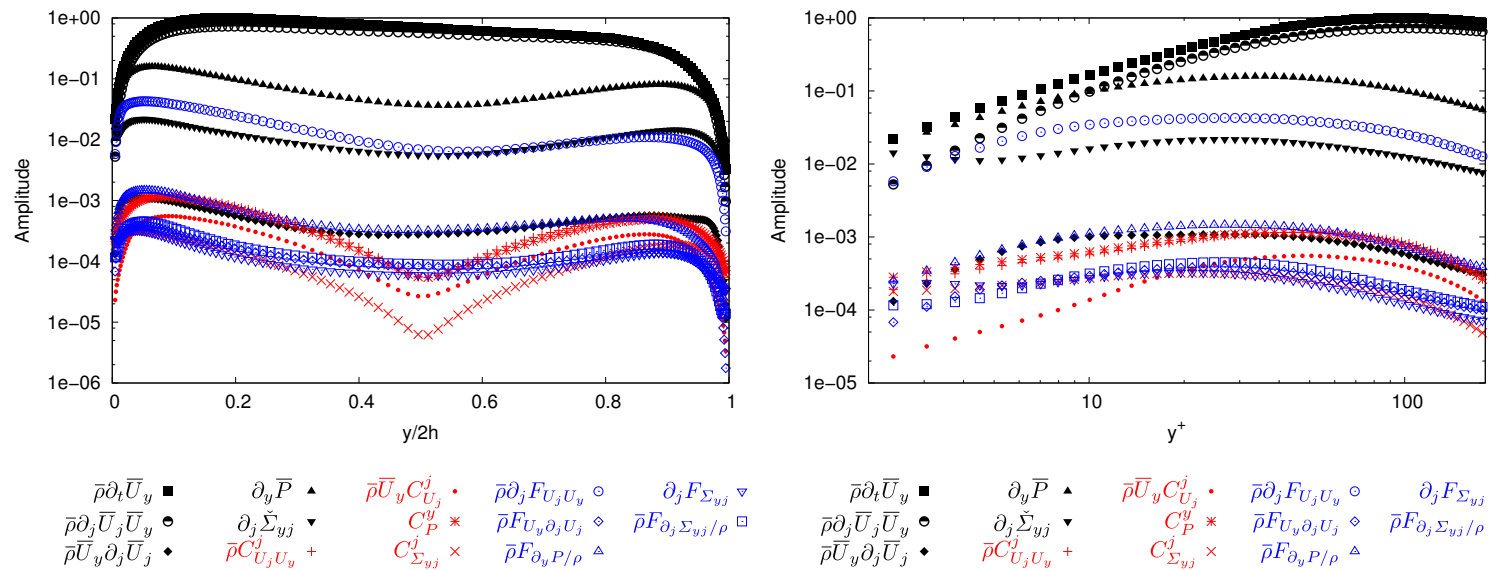

$\begin{array}{rrrrr}\bar{\rho} \partial_{t} \bar{U}_{y} \cdot & \partial_{y} \bar{P} \bullet & \bar{\rho} \bar{U}_{y} C_{U_{j}}^{j} \cdot & \bar{\rho} \partial_{j} F_{U_{j} U_{y}} \circ & \partial_{j} F_{\Sigma_{y j}} \nabla \\ \bar{\rho} \partial_{j} \bar{U}_{j} \bar{U}_{y} \bullet & \partial_{j} \bar{\Sigma}_{y j} \cdot & C_{P}^{y} * & \bar{\rho} F_{U_{y} \partial_{j} U_{j}} \diamond & \bar{\rho} F_{\partial_{j} \Sigma_{y j} / \rho} \square \\ \bar{\rho} \bar{U}_{y} \partial_{j} \bar{U}_{j} \cdot & \bar{\rho} C_{U_{j} U_{y}}^{j}+ & C_{\Sigma_{y j}}^{j} \times & \bar{\rho} F_{\partial_{y} P / \rho} \Delta & \end{array}$

(c) In the Velocity formulation.

Figure 7 - Root mean square of the terms of the wall-normal momentum conservation equation as a function of the wall-normal coordinate in the Classical formulation (a), in the Favre formulation (b) and of the terms of the wall-normal velocity transport equation and in the Velocity formulation (c) at $R e_{\tau}=180$ with the filter A. The amplitude is scaled by the maximum value in the domain in the three formulations. 

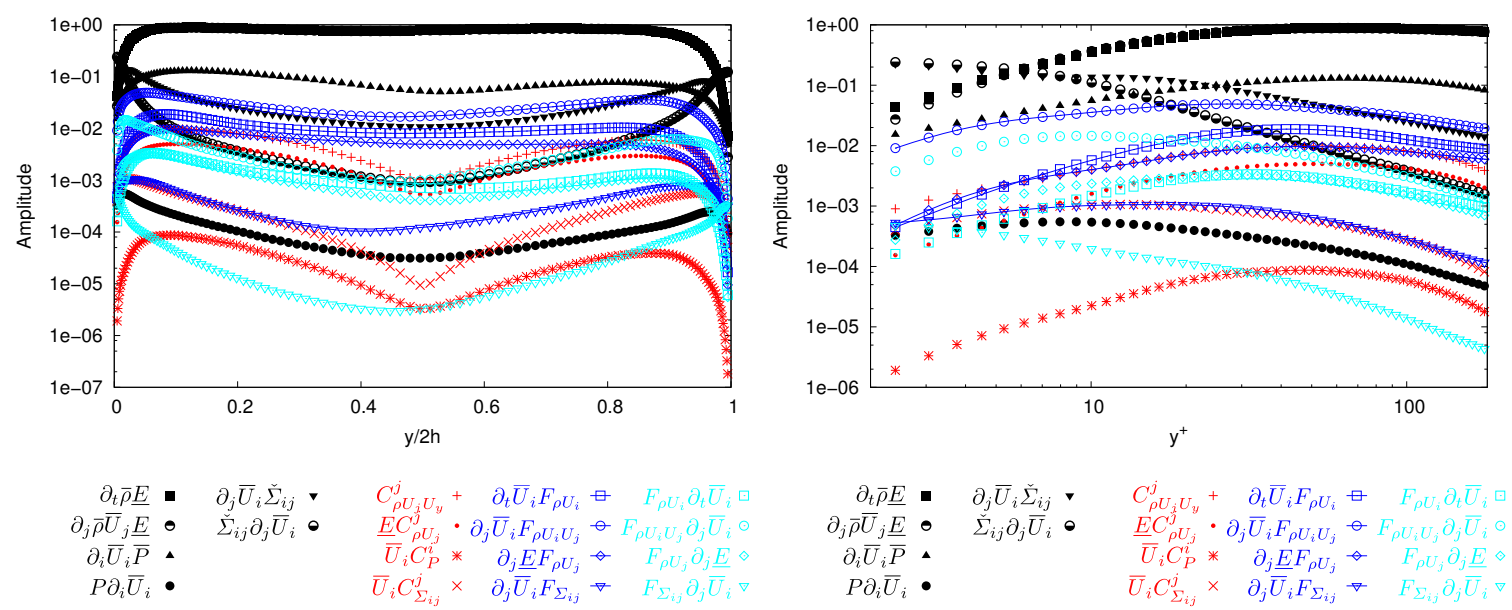

(a) In the Classical formulation.
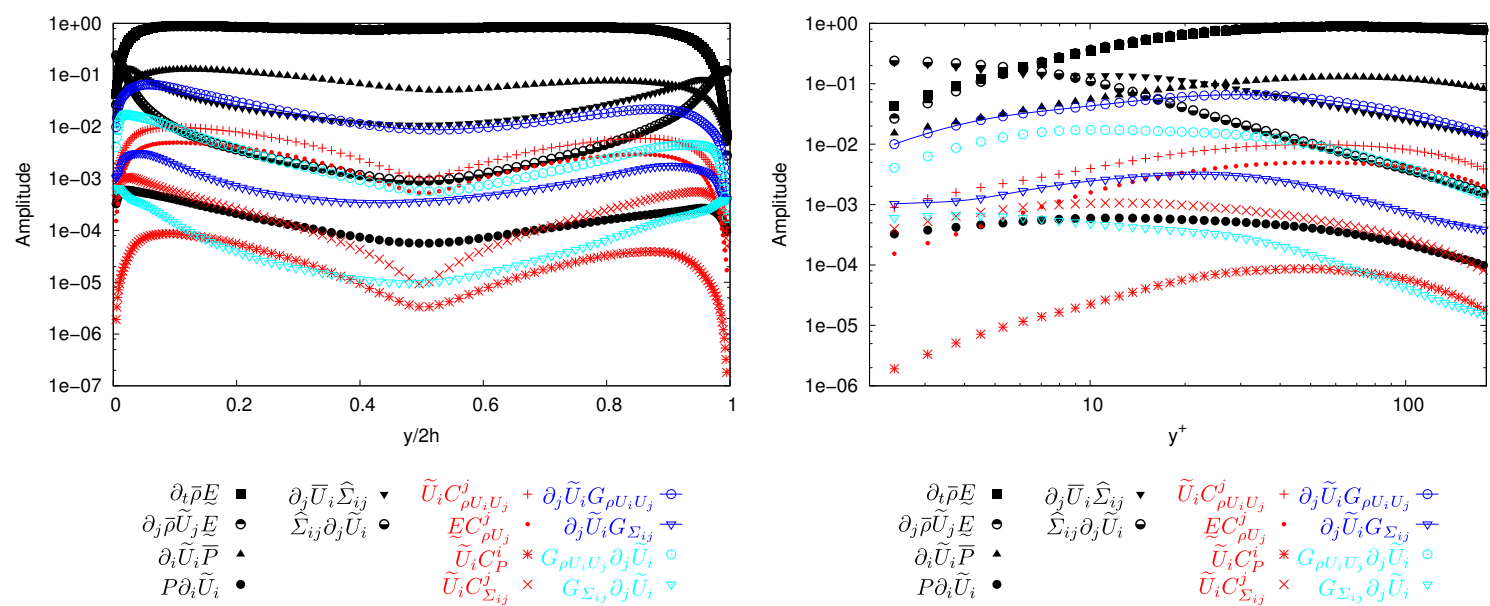

(b) In the Favre formulation.
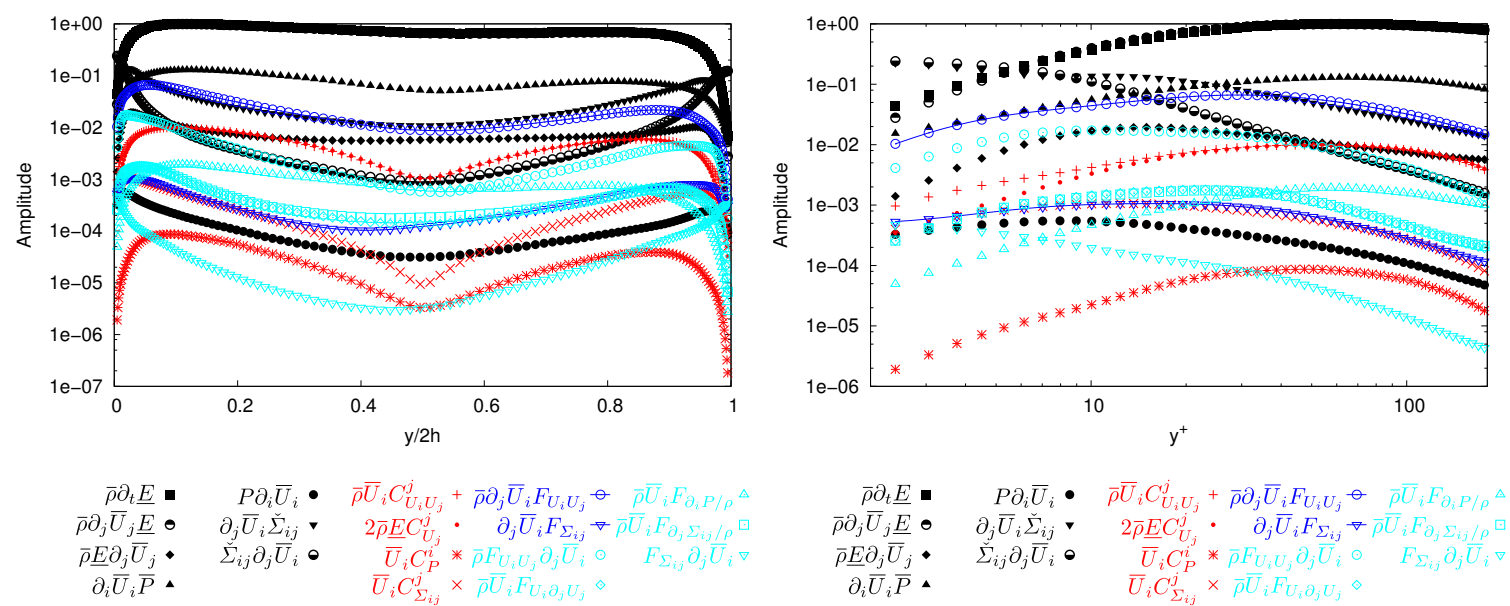

(c) In the Velocity formulation.

Figure 8 - Root mean square of the terms of the resolved kinetic energy transport equation as a function of the wall-normal coordinate in the Classical formulation (a), in the Favre formulation (b) and in the Velocity formulation (c) at $R e_{\tau}=180$ with the filter $\mathrm{A}$. The amplitude is scaled by the maximum value in the domain in the three formulations. 
equation. The velocity-dilatation product in the streamwise velocity transport equation is also small but is not negligible. This shows the small influence of dilatation on the flow dynamics in low Mach number strongly anisotermal flows. This is consistent with the negligible effect of dilatation on the turbulence kinetic energy budget [16].

In the momentum conservation equation (figures 5,6 and 7), the most significant subgrid term in the three formulations is the subgrid term associated with momentum or velocity convection, $\partial_{j} F_{\rho U_{j} U_{i}}, \partial_{j} \bar{\rho} G_{U_{j} U_{i}}$ and $\partial_{j} F_{U_{j} U_{i}}$. It is larger than viscous term in the bulk and cannot be neglected at the wall. This subgrid term has a larger amplitude at the centre of the channel in the Classical formulation than in the Favre and Velocity formulations but a slightly lower amplitude near the wall. It is harder to model in the Classical formulation since it is a triple correlation whereas it is a double correlation in the Favre and Velocity formulations.

Three subgrid terms have a medium-sized amplitude. In the Classical formulation, the time derivative subgrid term $\partial_{t} F_{\rho U_{i}}$ is the second most significant subgrid term. In particular, it has the same order of magnitude as the viscous term at the centre of the channel. Present in the three formulations, the filter-derivative noncommutation subgrid term associated with the convective term, $C_{\rho U_{j} U_{i}}^{j}$ or $C_{U_{j} U_{i}}^{j}$, has a lower amplitude than any non-negligible non-subgrid term but by less than one order of magnitude. It appears very small in the spanwise and wall-normal directions, but is more significant in the streamwise momentum equation. In the Velocity formulation, this subgrid term is followed by the velocity divergence filterderivative non-commutation subgrid term $C_{U_{j}}^{j}$, which has a similar behaviour. Since the subgrid terms $C_{U_{j} U_{i}}^{j}$ and $C_{U_{j}}^{j}$ appear with opposite sign in the velocity transport equation, the subgrid terms associated with the filter-derivative non-commutation are less significant in the Velocity formulation.

The subgrid term associated with the nonlinearities of the viscous diffusion, $\partial_{j} F_{\Sigma_{i j}}$ and $G_{\Sigma_{i j}}$, can be considered insignificant in the three formulations. Its amplitude is increased by around one order of magnitude in the Favre formulation but remains one order of magnitude smaller than any non-subgrid term in the entire channel. Assuming that the small-scale variations of velocity are significant, this suggests that the influence of small-scale variations of viscosity on the behaviour of the flow is negligible. The filter-derivative non-commutation subgrid term associated with the pressure and viscous terms $C_{P}^{i}$ and $C_{\Sigma_{i j}}^{i}$ are also negligible. All three of the additional subgrid terms in the Velocity formulation, $F_{U_{i} \partial_{j} U_{j}}, F_{\partial_{i} P / \rho}$ and $F_{\partial_{j} \Sigma_{i j} / \rho}$, are also found to have a very small amplitude. The most significant of the three is the subgrid term $F_{\partial_{i} P / \rho}$. The Velocity formulation thus appears more interesting than the classical formulation with regard to the subgrid-scale modelling as the time derivative subgrid term is replaced with three negligible subgrid terms.

The resolved kinetic energy transport equation (figure 9) confirms the classification of the subgrid terms in the momentum conservation equation. The most significant subgrid term in the momentum conservation equation have a significant energetic contribution, reaffirming the importance of the subgrid terms associated with momentum or velocity convection, $\partial_{j} F_{\rho U_{j} U_{i}}, \partial_{j} \bar{\rho} G_{U_{j} U_{i}}$ and $\partial_{j} F_{U_{j} U_{i}}$, and, in the Classical formulation, of the subgrid term associated with the time derivative term, 

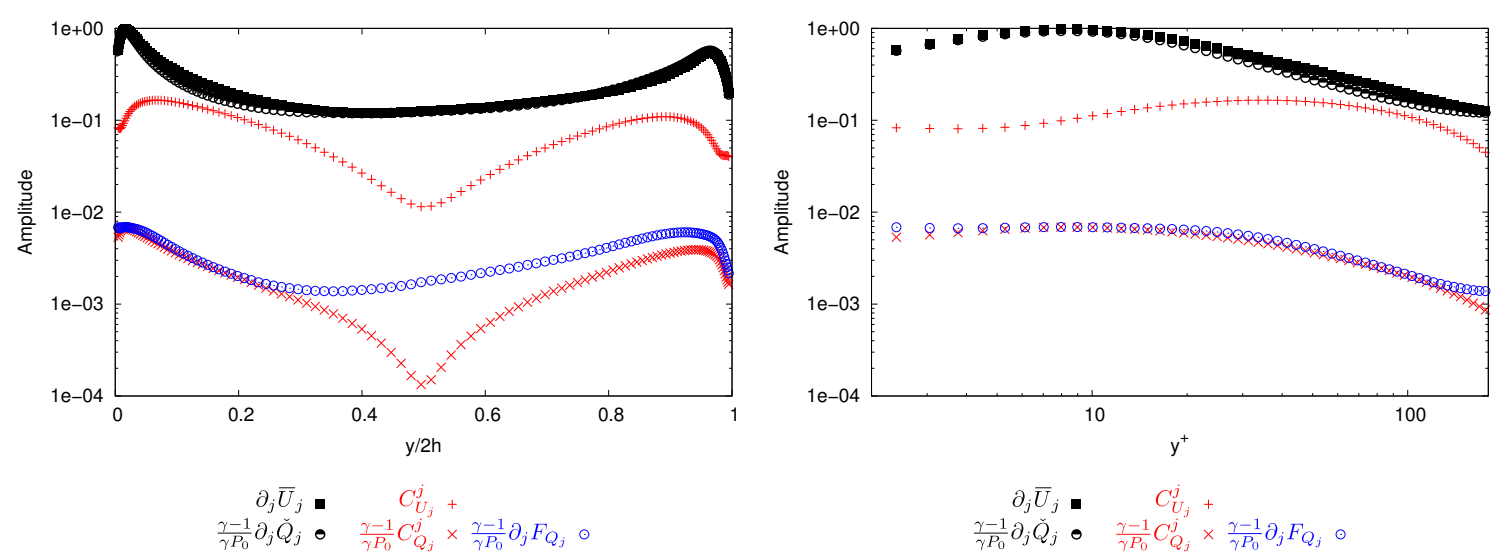

(a) With the classical filter.
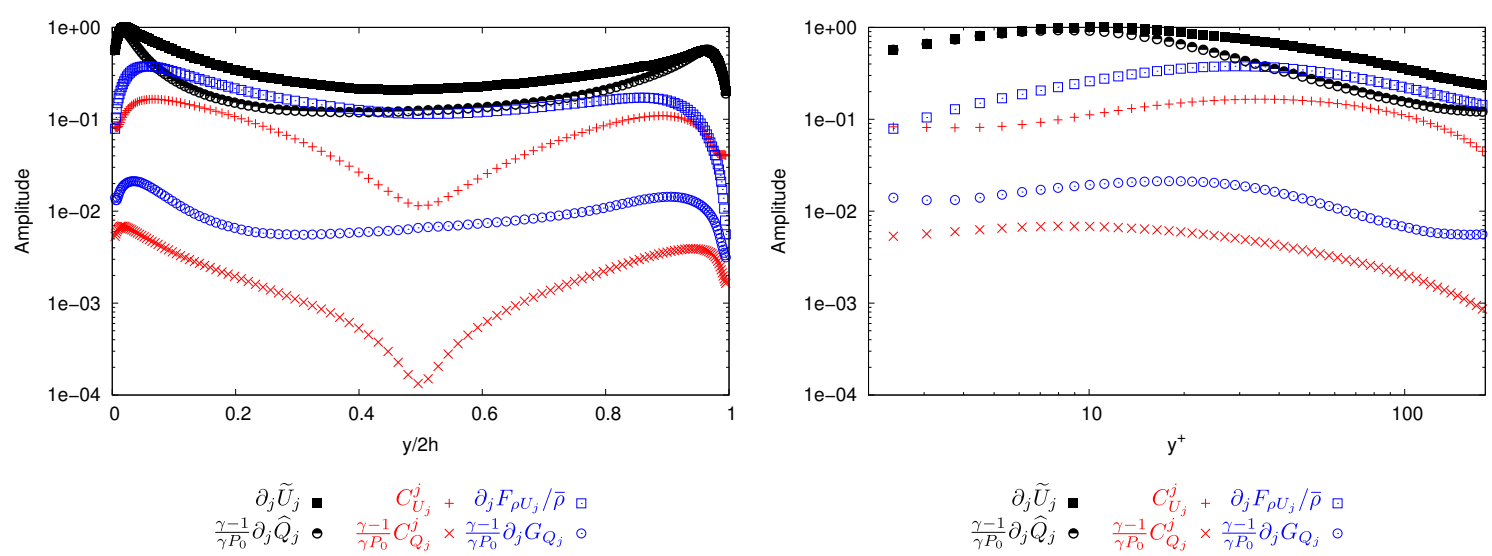

(b) With the Favre filter.

Figure 9 - Root mean square of the terms of the energy conservation equation as a function of the wall-normal coordinate in the Classical and Velocity formulations (a) and in the Favre formulation (b) at $R e_{\tau}=180$ with the filter A. The amplitude is scaled by the maximum value in the domain in the three formulations.

$\partial_{t} F_{\rho U_{i}}$.

\subsubsection{Energy conservation equation}

Omitting the time derivative of the thermodynamical pressure, constant in space, the energy conservation equation (figure 9) expresses the equality, up to a constant scalar factor, of the divergence of the velocity and of the heat flux. The difference between the divergence of the resolved velocity and heat flux represents the effect of the subgrid terms. With the classical filter (Classical or Velocity formulation), the only significant subgrid term is the filter-derivative non-commutation subgrid term $C_{U_{j}}^{j}$ associated with the divergence of the velocity. The subgrid terms $\partial_{j} F_{Q_{j}}$ and $C_{Q_{j}}^{j}$ associated with the heat flux are at least 50 times smaller than the non-subgrid terms throughout the entire channel. This is consistent with the assumption that the variations of conductivity over a small control volume can be neglected. With 

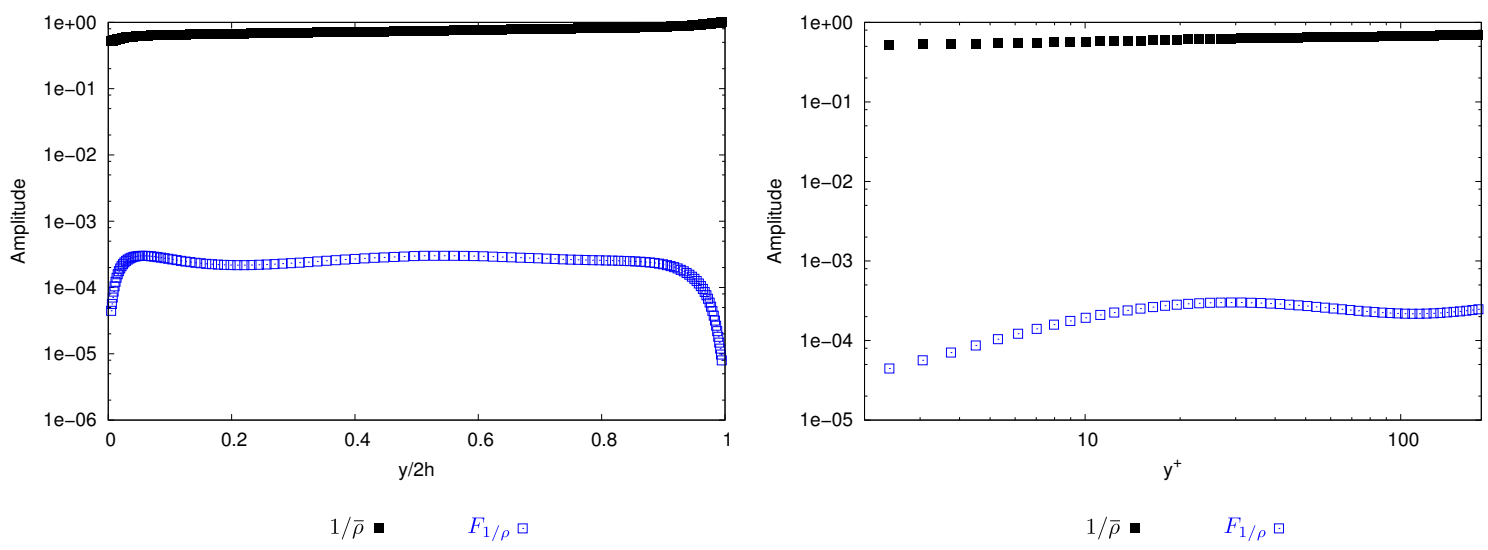

(a) With the classical filter.
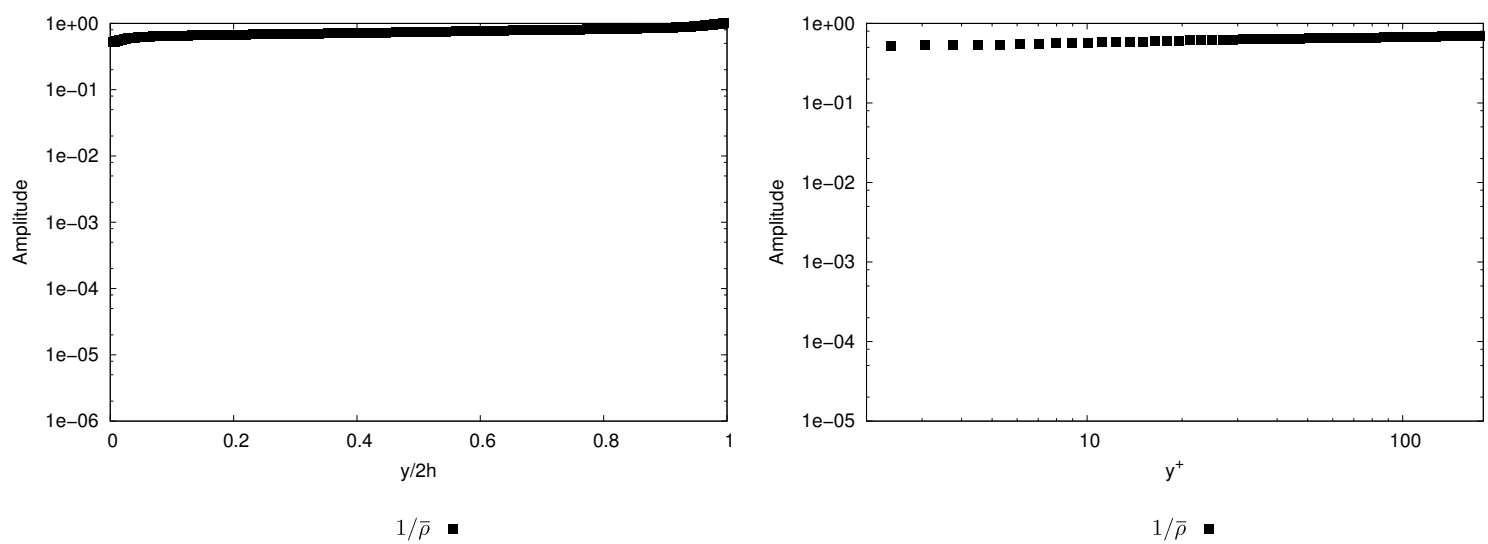

(b) With the Favre filter.

Figure 10 - Root mean square of the terms of the ideal gas law as a function of the wall-normal coordinate in the Classical and Velocity formulations (a) and in the Favre formulation (b) at $R e_{\tau}=180$ with the filter $\mathrm{A}$. The amplitude is scaled by the maximum value in the domain in the three formulations.

the Favre filter, the additional subgrid term associated with the density-velocity correlation $G_{U_{j} / \rho}$ has a large amplitude, of the same magnitude as the non-subgrid terms. In other words, the divergence of the Favre-filtered velocity is a poor approximation of the divergence of the velocity because of the small-scale variations of density and velocity. The density-velocity correlation is more significant in the energy conservation equation in the Favre formulation than in mass or momentum conservation equation in the Classical or Velocity formulations. The nonlinearity error $\partial_{j} G_{Q_{j}}$ associated with the heat flux is significantly larger with the Favre filter than with the classical filter but remains rather small.

\subsubsection{Ideal gas law}

The ideal gas law (figure 10) is used to compute the filtered temperature from the filtered density. With the Favre filter, there is theoretically no subgrid term in 


\begin{tabular}{|c|c|c|c|c|}
\hline Equation & Formulation & Large & Medium & Small or very small \\
\hline Mass & $\begin{array}{l}\text { Classical, Velocity } \\
\text { Favre }\end{array}$ & & $\begin{array}{c}\partial_{j} F_{\rho U_{j}}, C_{\rho U_{j}}^{j} \\
C_{\rho U_{j}}^{j}\end{array}$ & \\
\hline Momentum & $\begin{array}{l}\text { Classical } \\
\text { Favre } \\
\text { Velocity }\end{array}$ & $\begin{array}{l}\partial_{j} F_{\rho U_{j} U_{i}} \\
\partial_{j} \bar{\rho} G_{U_{j} U_{i}} \\
\bar{\rho} \partial_{j} F_{U_{j} U_{i}}\end{array}$ & $\begin{array}{c}\partial_{t} F_{\rho U_{i}}, C_{\rho U_{j} U_{i}}^{j} \\
C_{\rho U_{j} U_{i}}^{j} \\
\bar{\rho} C_{U_{j} U_{i}}^{j}, \bar{\rho} \bar{U}_{i} C_{U_{j}}^{j}\end{array}$ & $\begin{array}{c}C_{P}^{i}, \partial_{j} F_{\Sigma_{i j}}, C_{\Sigma_{i j}}^{i} \\
C_{P}^{i}, \partial_{j} G_{\Sigma_{i j}}, C_{\Sigma_{i j}}^{i} \\
\bar{\rho} F_{U_{i} \partial_{j} U_{j}}, \bar{\rho} F_{\partial_{i} P / \rho}, C_{P}^{i}, \\
\bar{\rho} F_{\partial_{j} \Sigma_{i j} / \rho}, \partial_{j} F_{\Sigma_{i j}}, C_{\Sigma_{i j}}^{i}\end{array}$ \\
\hline Energy & $\begin{array}{l}\text { Classical, Velocity } \\
\text { Favre }\end{array}$ & $G_{U_{j} / \rho}$ & $\begin{array}{l}C_{U_{j}}^{j} \\
C_{U_{j}}^{j}\end{array}$ & $\begin{array}{c}\partial_{j} F_{Q_{j}}, C_{Q_{j}}^{j} \\
\partial_{j} G_{Q_{j}}, C_{Q_{j}}^{j}\end{array}$ \\
\hline Ideal gas law & $\begin{array}{l}\text { Classical, Velocity } \\
\text { Favre }\end{array}$ & & & $F_{1 / \rho}$ \\
\hline
\end{tabular}

Table 1 - Classification of the subgrid terms in the three formulations.

the ideal gas law. With the classical filter (Classical or Velocity formulation), the subgrid term $F_{1 / \rho}$ it is found negligible. The ideal gas law can thus be used without model with both the classical and Favre filter.

\subsubsection{Summary}

The subgrid terms are classified according to their quadratic average in table 1. The large subgrid terms are the most significant subgrid terms, and should be modelled first. The medium subgrid terms have a smaller amplitude. The modelling of the small or very small subgrid terms should only be considered after all large and medium subgrid terms are modelled, and may not be recommended as their amplitude may not be larger than even the modelling error of the larger subgrid terms.

\subsection{Effect of the filter width}

The effect of the filter width on the subgrid term is investigated at $R e_{\tau}=180$ by comparing the amplitude of the subgrid terms with the filters A, B and C. As the filter size is increased, the magnitude of all subgrid term is increased compared to the non-subgrid terms. In the mass conservation equation, the momentum conservation equation and the ideal gas law, the increase is not sufficient to affect the conclusions of the classification of the subgrid terms, since the order of magnitude of the subgrid terms remains similar for the three filters. This supports the assumption that, while the variations across the channel of viscosity and thermal conductivity have a significant impact on turbulence [33], the effects of their small-scale variations on the flow are negligible.

In the energy conservation equation, the filter width has a strong influence on 
the magnitude of the filter-derivative non-commutation subgrid term $C_{U_{j}}^{j}$ associated with the divergence of the velocity. While the magnitude of this subgrid term is moderate with the filter A (figure 9), it is very large with the filter C (figure 11), as its amplitude is larger than that of the resolved heat flux from the logarithmic region to near the centre of the channel. The results with the filter B (not presented here) are in-between. The differences are primarily due to the increased anisotropy of the filter when the filter width is increased. The three filters follow a hyperbolic tangent law in the wall-normal coordinate direction, with a larger dilatation parameter the larger the filter width. With the filter $\mathrm{C}$, the subgrid term $C_{U_{j}}^{j}$ is rather small in the viscous sublayer and becomes very large farther from the wall. This suggests that a filter more uniform in the logarithmic region is preferable to limit the influence of this subgrid term.

The conclusions of the classification of the subgrid terms (1) are valid for the filter A and B. For the filter $\mathrm{C}, C_{U_{j}}^{j}$ is a large subgrid term. We consider its modelling necessary.

\section{Conclusion}

The low Mach number equations are suited to turbulent flows with a low Mach number but subjected to large variations of the fluid properties. They are characterised by a distinctive form of the energy conservation equation, that does not let us categorically choose between the classical filter and the Favre filter. In this study, we filter the low Mach number equations and identify the specific subgrid terms. Then, we investigate a priori the magnitude of all subgrid terms using the flow field from direct numerical simulations of a strongly anisothermal turbulent channel flow. The temperature gradient creates an asymmetry between the hot and cold sides regarding the amplitude and position of the maxima of the subgrid terms, explained by the local variations of the mean fluid properties and a low Reynolds number effect. Regardless of the formulation, more than half of the subgrid terms are found negligible. In particular, the effect of small-scale variations of viscosity and thermal conductivity may be neglected. The two most significant subgrid terms are the subgrid terms associated with momentum convection and with the density-velocity correlation. Due to the mesh inhomogeneity, the modelling of some filter-derivative non-commutation may also need to be considered, depending on the width of the selected filter. The classical filter is found more appropriate if the momentum equation is expressed as the velocity transport equation. The Favre filter removes the need for the modelling of the density-velocity correlation from the mass conservation equation but requires the modelling of an additional subgrid term in the energy equation, which has a very significant amplitude. The density-velocity correlation thus needs to be modelled in both cases. 

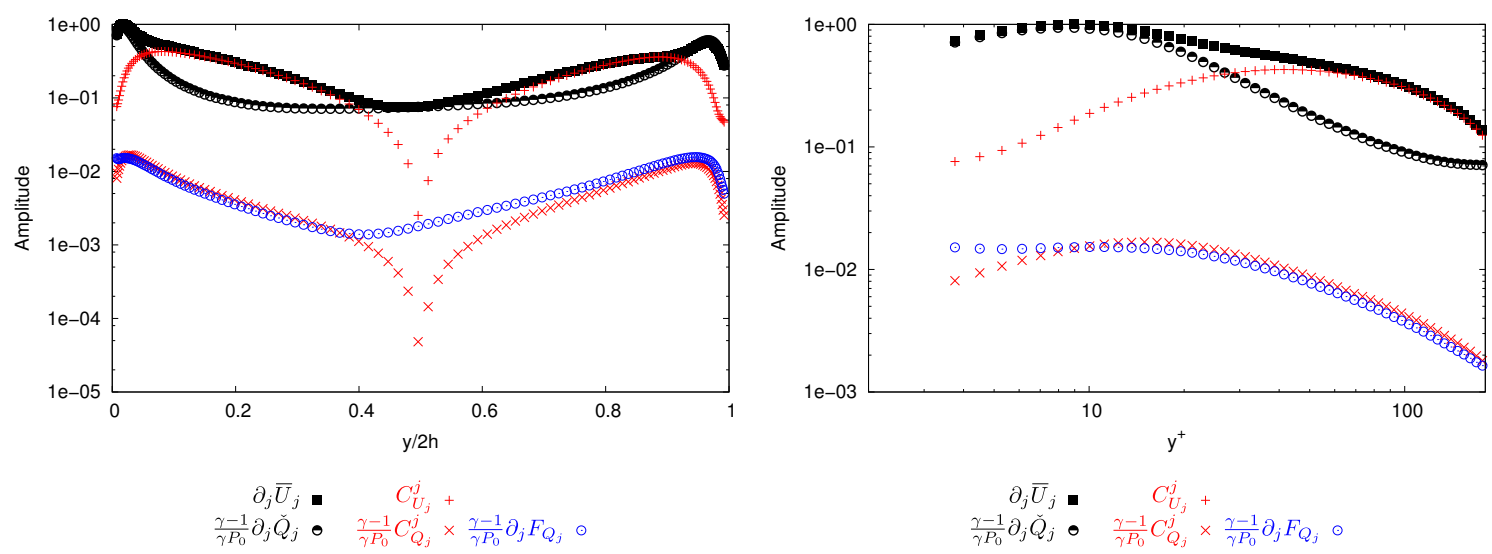

(a) With the classical filter.
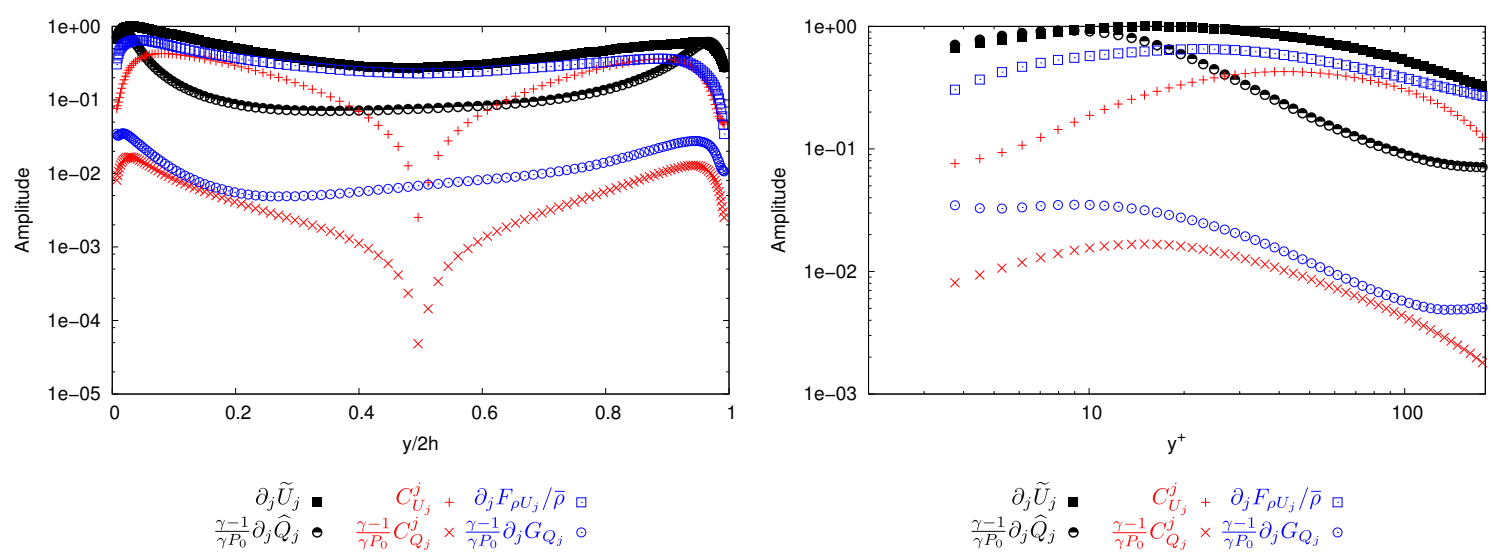

(b) With the Favre filter.

Figure 11 - Root mean square of the terms of the energy conservation equation as a function of the wall-normal coordinate in the Classical and Velocity formulations (a) and in the Favre formulation (b) at $R e_{\tau}=180$ with the filter C. The amplitude is scaled by the maximum value in the domain in the three formulations.

\section{Acknowledgment}

The authors gratefully acknowledge the CEA for the development of the TRUST platform. This work was granted access to the HPC resources of CINES under the allocations 2016-c20162a5099 and 2017-A0022A05099 made by GENCI.

\section{References}

[1] A. Abba, A. C. Cercignani, and L. Valdettaro. Analysis of subgrid scale models. Comput. Math. Appl., 46(4):521-535, 2003.

[2] N. Al-Zurfi and A. Turan. LES of rotational effects on film cooling effectiveness and heat transfer coefficient in a gas turbine blade with one row of air film injection. International Journal of Thermal Sciences, 99:96-112, 2016. 
[3] R. A. Antonia, M. Teitel, J. Kim, and L. W. B. Browne. Low-reynolds-number effects in a fully developed turbulent channel flow. Journal of Fluid mechanics, 236:579-605, 1992.

[4] F. Aulery, A. Toutant, F. Bataille, and Y. Zhou. Energy transfer process of anisothermal wall-bounded flows. Physics Letters A, 379(24):1520-1526, 2015.

[5] F. Aulery, D. Dupuy, A. Toutant, F. Bataille, and Y. Zhou. Spectral analysis of turbulence in anisothermal channel flows. Computers \& Fluids, 151:115-131, 2017.

[6] M. Bellec, A. Toutant, and G. Olalde. Large eddy simulations of thermal boundary layer developments in a turbulent channel flow under asymmetrical heating. Computers $\mathscr{G}$ Fluids, 151:159-176, 2017.

[7] D. J. Bodony and S. J. Lele. On using large-eddy simulation for the prediction of noise from cold and heated turbulent jets. Phys. Fluids, 17(8):085103, 2005.

[8] B. J. Boersma and S. K. Lele. Large eddy simulation of compressible turbulent jets. Center for Turbulence Research, Annual Research Briefs, pages 365-377, 1999.

[9] C. Calvin, O. Cueto, and P. Emonot. An object-oriented approach to the design of fluid mechanics software. ESAIM: Mathematical Modelling and Numerical Analysis, 36(05):907-921, 2002.

[10] M. A. Carper and F. Porté-Agel. Subfilter-scale fluxes over a surface roughness transition. part ii: A priori study of large-eddy simulation models. Boundary-Layer Meteorology, 127(1):73-95, 2008.

[11] S. C. P. Cheung and G. H. Yeoh. A fully-coupled simulation of vortical structures in a large-scale buoyant pool fire. International Journal of Thermal Sciences, 48(12): 2187-2202, 2009.

[12] S. G. Chumakov and C. J. Rutland. Dynamic structure subgrid-scale models for large eddy simulation. International Journal for Numerical Methods in Fluids, 47(8-9): 911-923, 2005.

[13] J. A. Clark. A study of incompressible turbulent boundary layers in channel flow. Journal of Basic Engineering, 90(4):455-467, 1968.

[14] R. A. Clark, J. H. Ferziger, and W. C. Reynolds. Evaluation of subgrid-scale models using an accurately simulated turbulent flow. J. Fluid Mech., 91(1):1-16, 1979.

[15] D. Dupuy, A. Toutant, and F. Bataille. Study of the sub-grid terms of the large-eddy simulation of a low Mach strongly anisothermal channel flow. In Eurotherm Seminar 106, Paris, France, 2016.

[16] D. Dupuy, A. Toutant, and F. Bataille. Turbulence kinetic energy exchanges in flows with highly variable fluid properties. Journal of Fluid Mechanics, 834:5-54, 2018.

[17] H. Eckelmann. The structure of the viscous sublayer and the adjacent wall region in a turbulent channel flow. Journal of Fluid Mechanics, 65(3):439-459, 1974.

[18] A. Favre. The equations of compressible turbulent gases. Technical Report AD0622097, DTIC Document, 1965.

[19] E. Garnier, N. Adams, and P. Sagaut. Large eddy simulation for compressible flows. Springer Science \& Business Media, 2009.

[20] N. S. Ghaisas and S. H. Frankel. A priori evaluation of large eddy simulation subgridscale scalar flux models in isotropic passive-scalar and anisotropic buoyancy-driven homogeneous turbulence. J. Turbulence, 15(2):88-121, 2014.

[21] H.-P. Kreplin and H. Eckelmann. Behavior of the three fluctuating velocity components in the wall region of a turbulent channel flow. The Physics of Fluids, 22(7): 1233-1239, 1979.

[22] C. Li. A-priori analysis of LES subgrid scale models applied to wall turbulence with pressure gradients. $\mathrm{PhD}$ thesis, Ecole Centrale de Lille, 2013. 
[23] W. Li, X. Li, J. Ren, and H. Jiang. Large eddy simulation of compound angle hole film cooling with hole length-to-diameter ratio and internal crossflow orientation effects. International Journal of Thermal Sciences, 121:410-423, 2017.

[24] S. Liu, C. Meneveau, and J. Katz. On the properties of similarity subgrid-scale models as deduced from measurements in a turbulent jet. J. Fluid Mech., 275:83-119, 1994.

[25] H. Lu, C. J. Rutland, and L. M. Smith. A priori tests of one-equation LES modeling of rotating turbulence. J. Turbulence, (8):N37, 2007.

[26] M. Pino Martín, U. Piomelli, and G. V. Candler. Subgrid-scale models for compressible large-eddy simulations. Theoretical and Computational Fluid Dynamics, 13(5):361$376,2000$.

[27] R. D. Moser, J. Kim, and N. N. Mansour. Direct numerical simulation of turbulent channel flow up to $\operatorname{Re}_{\tau}=590$. Phys. Fluids, 11(4):943-945, 1999.

[28] F. Nicoud. Conservative high-order finite-difference schemes for low-Mach number flows. J. Comput. Phys., 158(1):71-97, 2000.

[29] S. Paolucci. On the filtering of sound from the Navier-Stokes equations. Technical Report SAND82-8257, Sandia National Labs., Livermore, CA (USA), 1982.

[30] U. Piomelli, P. Moin, and J. H. Ferziger. Model consistency in large eddy simulation of turbulent channel flows. Phys. Fluids, 31(7):1884-1891, 1988.

[31] P. Sagaut. Large eddy simulation for incompressible flows: an introduction. Springer Science \& Business Media, 2006.

[32] M. Sanchez, F. Aulery, A. Toutant, and F. Bataille. Large eddy simulation of thermal boundary layer spatial development in a turbulent channel flow. Journal of Fluids Engineering, 136(6):060906, 2014.

[33] S. Serra, A. Toutant, and F. Bataille. Thermal large eddy simulation in a very simplified geometry of a solar receiver. Heat Transfer Engineering, 33(6):505-524, 2012.

[34] S. Serra, A. Toutant, F. Bataille, and Y. Zhou. High-temperature gradient effect on a turbulent channel flow using thermal large-eddy simulation in physical and spectral spaces. J. Turbulence, 13:N49, 2012.

[35] G. S. Sidharth and G. V. Candler. Stretched-vortex based subgrid-scale modeling for variable-density flows. In 45th AIAA Fluid Dynamics Conference, page 2782, 2015.

[36] G. S. Sidharth, A. Kartha, and G. V. Candler. Filtered velocity based LES of mixing in high speed recirculating shear flow. In 46th AIAA Fluid Dynamics Conference, page 3184, 2016.

[37] X.-B. Sun and X.-Y. Lu. A large eddy simulation approach of compressible turbulent flow without density weighting. Phys. Fluids, 18(11):118101, 2006.

[38] W. Sutherland. The viscosity of gases and molecular force. The London, Edinburgh, and Dublin Philosophical Magazine and Journal of Science, 36(223):507-531, 1893.

[39] A. Toutant and F. Bataille. Turbulence statistics in a fully developed channel flow submitted to a high temperature gradient. International Journal of Thermal Sciences, 74:104-118, 2013.

[40] A. Toutant, E. Labourasse, O. Lebaigue, and O. Simonin. DNS of the interaction between a deformable buoyant bubble and a spatially decaying turbulence: a priori tests for LES two-phase flow modelling. Computers $\&$ Fluids, 37(7):877-886, 2008.

[41] A. W. Vreman and J. G. M. Kuerten. Comparison of direct numerical simulation databases of turbulent channel flow at $\operatorname{Re}_{\tau}=180$. Phys. Fluids, 26(1):015102, 2014.

[42] B. Vreman. Direct and large-eddy simulation of the compressible turbulent mixing layer. PhD thesis, University of Twente, 1995.

[43] B. Vreman, B. Geurts, and H. Kuerten. A priori tests of large eddy simulation of the 
compressible plane mixing layer. Journal of Engineering Mathematics, 29(4):299-327, 1995.

[44] B. Vreman, B. Geurts, and H. Kuerten. Subgrid-modelling in LES of compressible flow. Applied Scientific Research, 54(3):191-203, 1995.

[45] S. M. Yahya, S. F. Anwer, and S. Sanghi. Turbulent forced convective flow in an anisothermal channel. International Journal of Thermal Sciences, 88:84-95, 2015.

[46] A. Yoshizawa. Statistical theory for compressible turbulent shear flows, with the application to subgrid modeling. Phys. Fluids, 29(7):2152-2164, 1986. 